\section{A quantitative mapping approach to identify direct interactions within complexomes}

Philipp Trepte ${ }^{1,2, *, \#}$, Christopher Secker ${ }^{1, \#}$, Soon Gang Choi ${ }^{3,4,5, \#}$, Julien Olivet ${ }^{3,4,5,6, \#,}$ Eduardo Silva Ramos ${ }^{1}$, Patricia Cassonnet ${ }^{7}$, Sabrina Golusik1, Martina Zenkner ${ }^{1}$, Stephanie Beetz ${ }^{1}$, Marcel Sperling ${ }^{8}$, Yang Wang ${ }^{3,4,5}$, Tong Hao ${ }^{3,4,5}$, Kerstin Spirohn ${ }^{3,4,5}$, Jean-Claude Twizere ${ }^{6}$, Michael A. Calderwood ${ }^{3,4,5}$, David E. Hill ${ }^{3,4,5}$, Yves Jacob ${ }^{3,7}$, Marc Vidal $^{3,4, *}$, Erich E. Wanker ${ }^{1, *}$

${ }^{1}$ Neuroproteomics, Max Delbrück Center for Molecular Medicine, Robert-Rössle-Str. 10, 13125, Berlin, Germany. ${ }^{2}$ Brain Development and Disease, Institute of Molecular Biotechnology of the Austrian Academy of Sciences (IMBA), Dr. Bohr-Gasse 3, 1030, Vienna, Austria.

${ }^{3}$ Center for Cancer Systems Biology (CCSB), Dana-Farber Cancer Institute (DFCI), 450 Brookline Avenue, Boston, MA, 02215, USA.

${ }^{4}$ Department of Genetics, Blavatnik Institute, Harvard Medical School (HMS), 77 Avenue Louis Pasteur, Boston, MA, 02115, USA.

${ }^{5}$ Department of Cancer Biology, Dana-Farber Cancer Institute (DFCI), 450 Brookline Avenue, Boston, MA, 02215, USA.

${ }^{6}$ Laboratory of Viral Interactomes, Unit of Molecular Biology of Diseases, Interdisciplinary Cluster for Applied Genoproteomics (GIGA Institute), University of Liège, 1 Avenue de l'hopital, 4000, Liège, Belgium.

${ }^{7}$ Département de Virologie, Unité de Génétique Moléculaire des Virus à ARN (GMVR), Institut Pasteur, UMR3569, Centre National de la Recherche Scientifique (CNRS), Université de Paris, 28 rue du Docteur Roux, 75015, Paris, France.

${ }^{8}$ Multifunctional Colloids and Coating, Fraunhofer Institute for Applied Polymer Research (IAP), Geiselbergstr. 69, 14476 Potsdam-Golm, Germany.

\#These authors contributed equally: Philipp Trepte, Christopher Secker, Soon Gang Choi, Julien Olivet.

*Correspondence should be addressed to: philipp.trepte@imba.oeaw.ac.at; marc_vidal@dfci.harvard.edu; erich.w@mdc-berlin.de 
ABSTRACT

Complementary methods are required to fully characterize all protein complexes, or the complexome, of a cell. Affinity purification coupled to mass-spectrometry (AP-MS) can identify the composition of complexes at proteome-scale. However, information on direct contacts between subunits is often lacking. In contrast, solving the 3D structure of protein complexes can provide this information, but structural biology techniques are not yet scalable for systematic, proteome-wide efforts. Here, we optimally combine two orthogonal highthroughput binary interaction assays, LuTHy and $\mathrm{N} 2 \mathrm{H}$, and demonstrate that their quantitative readouts can be used to differentiate direct interactions from indirect associations within multiprotein complexes. We also show that LuTHy allows accurate distance measurements between proteins in live cells and apply these findings to study the impact of the polyglutamine expansion mutation on the structurally unresolved $\mathrm{N}$-terminal domain of Huntingtin. Thus, we present a new framework based on quantitative interaction assays to complement structural biology and AP-MS techniques, which should help to provide first-approximation contact maps of multiprotein complexes at proteome-scale.

47 


\section{INTRODUCTION}

Characterizing the molecular architecture of all protein complexes, or the complexome, of a cell is essential to fully decipher its biological functions ${ }^{1,2}$. Protein complexes vary in sizes and compositions as they often consist of different types of macromolecules including DNA, RNA and lipids. For example, in human cells, $\sim 80$ proteins closely assemble with different ribosomal RNAs to form a functional ribosome, which is a compact spherical particle with a diameter of 250 to $300 \AA^{3,4}$. In comparison, the human proteasome is composed of eight protein subunits that co-assemble into a stable cylindrical complex ${ }^{5}$. This exemplifies how structurally diverse proteins can dynamically assemble in cells to perform various biological functions.

Affinity purification coupled to mass spectrometry (AP-MS) techniques are highly efficient in identifying the composition of protein complexes at proteome-scale ${ }^{6-9}$, but their ability to elucidate direct contacts between potentially interacting subunits is limited ${ }^{10,11}$. However, gaining information on direct interactions, i.e. on proteins sharing a physical interface, and indirect associations, i.e. proteins that do not directly interact, within complexes is important to understand their biological roles. For example, clearly defining interacting domains between proteins will help in the design of hypothesis-driven functional studies. Additionally, defining the relationship between complex members is crucial to characterize the effect of disease-associated missense mutations on protein assemblies, or for the identification and validation of drug targets.

Structural biology technologies such as X-ray diffraction (XRD), nuclear magnetic resonance (NMR), or cryo-electron microscopy (cryo-EM) provide high-resolution data on the assembly of multiprotein complexes. This includes information on the folding of protein domains, amino acid composition at contact sites, attachment of compounds to proteins, and post-translational modifications of specific amino acids. However, in comparison to highthroughput AP-MS-based methods, generation of protein interaction data using structural biology techniques can be labor intensive and time consuming. In addition, such techniques 
often use truncated instead of full-length proteins, and comprehensive, high-resolution

77 structural information is still missing for most protein complexes. Indeed, based on a recent study by Drew et al, $\sim 7,000$ human protein complexes can be found in the human proteome from the integration of over 15,000 published mass-spectrometry experiments ${ }^{12,13}$. However, only $\sim 4 \%$ of those (i.e. 309 protein complexes) currently have a resolved structure in the literature. Thus, complementary techniques are required to precisely characterize protein complexes currently identified by AP-MS-based methods ${ }^{7,11,14}$. revealed that the yeast two-hybrid $(\mathrm{Y} 2 \mathrm{H})$ system preferentially detects directly interacting proteins $^{10,15}$, and can thus provide a first-approximation view of the overall organization of complexes. While relatively versatile and amenable to high-throughput strategies, binary interaction assays are somewhat limited by their sensitivity ${ }^{16,17}$. We have recently demonstrated that this limitation can be overcome by optimally combining complementary assays and/or versions thereof to increase sensitivity without affecting specificity ${ }^{18,19}$.

Here we investigate a new combination of two binary interaction assays, the bioluminescence-based two-hybrid (LuTHy) method ${ }^{19}$, and the mammalian cell expression version of the NanoLuc two-hybrid $(\mathrm{mN} 2 \mathrm{H})$ assay $^{18}$ to systematically map pairwise interactions between subunits of three multiprotein complexes, LAMTOR, BRISC and MIS12, for which both $\mathrm{AP}-\mathrm{MS}^{7,11,14}$ and high-resolution structural data are available ${ }^{20-22}$. We find that direct interactions confer significantly higher scores than indirect associations, which allows their differentiation. Finally, we provide experimental evidence that the bioluminescence resonance energy transfer (BRET) readout of LuTHy can be used as a molecular ruler in live cells to estimate distances between protein domains for which no high-resolution structure is currently available. Our combined quantitative PPI mapping approach should thus be applicable for the in-depth characterization of subunit interactions at complexome-scale. 


\section{RESULTS}

\section{Benchmarking LuTHy under maximized specificity}

We previously demonstrated that combining multiple complementary interaction assays and/or versions thereof significantly increases PPI recovery ${ }^{17-19}$. Here, we first benchmarked the PPI detection performance of LuTHy, a bioluminescence-based doublereadout technology ${ }^{19}$, against an established positive reference set (PRS), hsPRS-v2, which contains 60 well-characterized human PPIs ${ }^{17,18}$. To control for specificity, a random reference set (RRS), hsRRS-v2, made of 78 pairs of human proteins not known to interact, was also tested $^{17,18}$. With LuTHy, the interaction between two proteins of interest, $X$ and $Y$, is first measured in living cells by quantification of the BRET signal (LuTHy-BRET; Supplementary Figure 1A). Then, following cell lysis, the same interaction is assessed in vitro by a quantitative luminescence-based co-precipitation readout (LuTHy-LuC; Supplementary

Figure 1A). Since LuTHy plasmids allow expression of each protein as $\mathrm{N}$ - or C-terminal fusions, and as donor (NL; NanoLuc tag) or acceptor (mCit; mCitrine tag) proteins, eight tagging configurations can be assessed for every protein pair of interest (Supplementary

Figure 1B). Thus, when all eight configurations are tested, a typical LuTHy experiment with both BRET and LuC measurements generates a total of 16 data points (Figure 1A,B; Source Data Figures 1-2).

PPI detection in hsPRS-v2 by LuTHy was measured under conditions of maximal specificity, i.e. under conditions where none of the random protein pairs from hsRRS-v2 are scored positive (Supplementary Figure 1C). In total, using all eight tagging configurations with both readouts (i.e. BRET and LuC), LuTHy identified 43 of the 60 PPIs tested in hsPRSv2, i.e. $71.7 \%$ (Figure 1C-E), confirming our previous observation that binary PPI mapping can be maximized when multiple assay versions are combined ${ }^{18,19}$.

As reported in Choi et $\mathrm{al}^{18}$, under identical conditions of maximal specificity, the union of 16 versions of five distinct PPI assays, GPCA, KISS, MAPPIT, Y2H and NanoBiT, identified 
of $60.0 \%$ (Figure 1E; Supplementary Figure 2A,B). Thus, LuTHy, with its double readout system, is a highly sensitive and specific PPI detection method that recovers most of the wellestablished hsPRS-v2 interactions.

\section{Detecting direct interactions supported by high-resolution structural data}

To further evaluate the extent to which different binary PPI assays can detect directly interacting proteins, we compared the recovery of the 31 hsPRS-v2 PPIs for which highresolution structures are available in the RCSB protein data bank (PDB) ${ }^{23}$ (hsPRS-PDB), to that of the 29 remaining interactions (hsPRS-non-PDB) (Figure 2A). Our analysis revealed that structurally supported PPIs are detected with higher success rates in comparison to interactions for which structural information is not currently available (Figure 2B). This was the case for: 1) our previous benchmarking of five binary assays combined (Five assays union, $80.6 \%$ hsPRS-PDB vs. $48.3 \%$ hsPRS-non-PDB); 2 ) the union of $12 \mathrm{~N} 2 \mathrm{H}$ assay versions (N2H union, $71.0 \%$ vs. $48.3 \%$ ); and 3) the newly benchmarked LuTHy method (LuTHy union, $80.6 \%$ vs. $62.1 \%$ ) (Figure 2B). Again, we confirmed our previous finding that the success of

142 interaction detection significantly increases when multiple complementary PPI detection assays, or assay versions, are used. Indeed, we found that combining all seven PPI assays (GPCA, KISS, MAPPIT, Y2H, NanoBiT, N2H and LuTHy) allowed recovering 30 of the 31 structurally supported PPIs (i.e. 96.8\%) under conditions of maximal specificity (Union all assays, Figure 2B,C). This demonstrates that structurally supported, direct PPIs can be recovered with nearly complete success rate when combining currently available binary PPI assays.

\section{Systematically mapping interactions within distinct multiprotein complexes}

To generalize these findings, we decided to map PPIs between subunits of wellcharacterized human multiprotein complexes. We selected three complexes based on the following criteria: 1) identified by AP-MS-based methods ${ }^{7,11,14}$; 2) contains at least four subunits; 3 ) at least one 3D structure available in $\mathrm{PDB}^{23}$; and 4 ) at least $80 \%$ of cloned open 
reading frames (ORFs) encoding the reported subunits present in the human ORFeome 8.1 collection ${ }^{24}$. This resulted in a list of 24 distinct protein complexes (Supplementary Table 1), among which three structurally diverse candidates with well-characterized biological functions were prioritized: 1) the LAMTOR complex, also termed "Ragulator" complex ${ }^{20}$, which regulates MAP kinases and mTOR activities and consists of seven subunits (LAMTOR1, LAMTOR2, LAMTOR3, LAMTOR4, LAMTOR5, RRAGA and RRAGC); 2) the MIS12 complex that connects the kinetochore to microtubules ${ }^{21}$, and is made of five subunits (CENPC1, DSN1, MIS12, NSL1 and PMF1); and 3) the BRISC complex, a large deubiquitinating machinery ${ }^{22}$ consisting of five proteins (ABRAXAS2, BABAM1, BABAM2, BRCC3 and SHMT2)

To map interactions between subunits of the LAMTOR, MIS12 and BRISC complexes, we systematically tested all possible pairwise combinations with the LuTHy and mN2H assays (Source Data Figures 3-5). Except for LAMTOR5, which was not available in the human ORFeome 8.1 collection, all 16 ORFs encoding the selected target proteins were sequence verified and cloned into both LuTHy and $\mathrm{N} 2 \mathrm{H}$ expression plasmids. A resulting search space of 256 pairwise combinations, corresponding to a total of 16 subunits for the three complexes (16x16 matrix; Figure 3B), was thus systematically explored with the LuTHy and mN2H assays. To score a tested interaction as positive, we rationalized that true binary PPIs should only be found between the respective subunits of a given complex (i.e. intra-complex pairs), but not between subunits belonging to different complexes (i.e. inter-complex pairs). Therefore, we treated all inter-complex pairs as negative controls, similar to protein pairs from a random reference set. We observed that LuTHy and $\mathrm{mN} 2 \mathrm{H}$ fusion constructs showed a broad distribution of interaction scores among those inter-complex pairs (Supplementary

177 Figure 3A-F). Therefore, we defined construct-specific cutoffs under conditions of maximal specificity, where none of the inter-complex protein pairs are scored positive with any of the assay versions. Using this strategy, we found that LuTHy and $\mathrm{mN} 2 \mathrm{H}$ recovered $35(68.6 \%)$ and $23(45.1 \%)$ of the 51 intra-complex interactions, respectively, while 42 of those protein 
182 Figure 4). We then compared those results to the ones from the BioPlex dataset ${ }^{7,11,14}$.

183 According to BioPlex, $66.7 \%$ of all intra-complex interactions but none of the inter-complex

184 protein pairs for the LAMTOR, BRISC and MIS12 complexes were detected by AP-MS

185 (Figure 3C). This demonstrates that, similar to AP-MS-based techniques, binary PPI mapping 186 with LuTHy and $\mathrm{mN} 2 \mathrm{H}$ can recover a large fraction of interactions between subunits of distinct multiprotein complexes with high sensitivity and specificity.

\section{Differentiating direct interactions from indirect associations}

Differentiating direct interactions from indirect associations within each multiprotein complex identified by AP-MS-based methods is an important, unresolved challenge. In fact, it was estimated that only $\sim 32 \%$ of the detected protein associations in the BioPlex dataset are direct interactions ${ }^{11}$. Therefore, we evaluated if the binary PPI detection assays, LuTHy and $\mathrm{mN} 2 \mathrm{H}$, could identify directly interacting subunits within multiprotein complexes. To define direct interactions and indirect associations between the subunits of each complex, we used $3 \mathrm{D}$ structural information available in three public databases: $\mathrm{PDB}^{23}$, Interactome $3 \mathrm{D}^{25}$ and PDBePISA ${ }^{26}$. While directly interacting proteins share a physical interface, we defined indirect associations as pairs of subunits that do not share an interaction interface (Figure 4A). In total, we classified 31 direct interactions and 7 indirect associations between the subunits of the LAMTOR, BRISC and MIS12 complexes. Noticeably, we excluded 13 separate homodimer interactions from all further analyses since they were not reported in the available multiprotein complex structures (Supplementary Table 2). When applying the same cutoffs used to differentiate intra- from inter-complex protein pairs, $70.9 \%, 45.2 \%$, and $48.4 \%$ of the direct interactions could be detected with LuTHy-BRET, LuTHy-LuC, and $\mathrm{mN} 2 \mathrm{H}$, respectively (Supplementary Figure 5A). While mN2H only detected direct interactions, LuTHy-BRET and LuTHy-LuC also recovered $71.4 \%$ and $28.6 \%$ of the indirect associations, respectively (Supplementary Figure 5B). The union of LuTHy and mN2H detected a total of $80.6 \%$ of the structure-based direct interactions, and $85.7 \%$ of the indirect associations (Supplementary

Figure 5A,B). In comparison, according to the BioPlex dataset, $87.1 \%$ of the direct 
interactions along with $100 \%$ of the indirect associations were recovered by AP-MS

\section{(Supplementary Figure 5A,B).}

To further evaluate if we could confidently differentiate between direct interactions and

212 indirect associations, we analyzed the quantitative scores obtained with LuTHy and mN2H.

213 We observed that direct interactions generate significantly higher scores compared to indirect associations in the LuTHy-BRET (Figure 4B), LuTHy-LuC (Figure 4C) and mN2H (Figure 4D) assays. Since LuTHy recovered both direct interactions and indirect associations, we applied a machine learning-based clustering algorithm on the LuTHy-BRET and LuTHy-LuC scores to classify LuTHy-positive protein pairs as direct or not-direct interactions. We used the detected hsPRS-PDB PPIs as a positive training set for direct interactions, and the hsRRS-v2 pairs as a negative training set for not-direct interactions (Supplementary Table 4). Next, we applied the trained cluster algorithm to the 23 direct interactions and six indirect associations detected by LuTHy (Supplementary Figure 5A,B). Using this unbiased approach, we were able to classify 14 of the LuTHy-positive direct interactions (45.2\%) as true direct PPIs, without wrongly classifying any of the indirect associations (Figure 4E,F, Supplementary Figure 5C). After combining these results with the ones from the $\mathrm{mN} 2 \mathrm{H}$ assay, we were able to confidently recover and classify $67.7 \%$ of the direct interactions among the LAMTOR, BRISC and MIS12 complexes. In comparison, protein pair associations reported in the BioPlex database are currently neither classified as direct PPIs or indirect associations (Figure 4F, Supplementary

Figure $\mathbf{5 C})^{7,11,14}$.

To better understand why a broad range of quantitative scores is obtained when the same pair of directly interacting proteins is tested in eight different tagging configurations (Supplementary Figure 6A-I), we explored the structural features of all direct interactions among the three complexes. Using PDBePISA ${ }^{26}$ we obtained the sizes of interaction interfaces as well as the total surface areas for all directly interacting subunits within the LAMTOR, BRISC and MIS12 complexes (Supplementary Table 5). We observed a stronger correlation between interaction scores and the sizes of the interaction interfaces, rather than to the total surface areas of the protein pairs (Figure 4G; Supplementary Figure 7A-F), 
indicating that the larger the interaction interface, i.e. the stronger the binding ${ }^{27}$, the higher the interaction score. Noticeably, LuTHy-BRET showed relatively high corrected BRET (cBRET) ratios for small interaction interfaces $\left(<500 \AA^{2}\right.$, Supplementary Figure $\left.7 A\right)$, which supports our previous findings that low affinity interactions can be detected with this assay ${ }^{19}$.

241 Surprisingly, a significant negative correlation between cBRET ratios and the total surface areas of protein pairs was observed (Figure 4G; Supplementary Figure 7D-F), suggesting that the larger the co-complex, the lower the resonance energy transfer between the $\mathrm{N}$ - or $\mathrm{C}$ -

244 terminally fused NanoLuc luciferase and mCitrine tags. This indicates that both affinity and 245 distance between the tagged proteins studied with binary PPI assays can be determinants for the intensity of the quantitative scores, and thus for their detection.

To test whether the LuTHy-BRET readouts could be used to measure distances between protein domains, we determined the molecular distances between all protein termini among the three complexes using available structural information (Source Data Figures 35). We then compared those reference values to the measured cBRET ratios and found a significant negative correlation between them and the molecular distances of the protein termini (Supplementary Figure 7G). Interestingly, no such relationship could be observed for the non-energy transfer-based corrected $\mathrm{LuC}(\mathrm{cLuC})$ or $\mathrm{mN} 2 \mathrm{H}$ ratios (Supplementary Figure $\mathbf{7 H}, \mathbf{I})$. In addition, the distances between protein termini of indirect associations identified with LuTHy-BRET were found to be within a similar range of distances than that of direct interactions (Supplementary Figure $7 \mathbf{J}$ ). This suggests that LuTHy-BRET can detect indirect associations between proteins even in the absence of a common interaction interface as long as the tagged proteins are within a distance where an energy transfer between the donor and the acceptor can occur.

Altogether, these results show that the quantitative scores obtained with LuTHy and $\mathrm{mN} 2 \mathrm{H}$ can be used to systematically identify direct interactions within multiprotein complexes and differentiate them from indirect associations. They also highlight that the measured CBRET ratios are correlated to the molecular distances between the protein termini of directly 
interacting subunits, which implies that LuTHy-BRET measurements could also be used to measure distances directly in live cells.

\section{Measuring molecular distances between subunits of protein complexes in live cells}

Förster resonance energy transfer-based techniques have been previously used as spectroscopic rulers ${ }^{28,29}$ to monitor conformational changes in proteins, or to estimate the proximity relationships of macromolecules in complexes ${ }^{30-32}$. Since high-resolution structural data are currently unavailable or incomplete for many protein complexes ${ }^{13}$, we investigated whether the LuTHy-BRET readout could be used to measure distances between full-length subunits of protein complexes in live cells. To establish a cBRET-distance standard curve, we used the structure-based molecular distances, the cBRET ratios of directly interacting subunits in the LAMTOR, BRISC and MIS12 complexes, and the structurally supported hsPRS-v2 PPIs (i.e. hsPRS-PDB) as references (Supplementary Figure 8A, Source Data Figures 1-2 and 3-5). However, many of these protein pairs are, on average, structurally covered by less than $75 \%$ of their respective full-length sequences (Supplementary Figures 8B and 9;

Supplementary Tables 5 and 6, Source Data Figures 3-5). Therefore, for the calibration, we selected a subset of 44 protein pairs (i.e. eight different interactions in multiple taggingconfigurations) where the tagged protein termini are fully resolved in the associated structures. For this subset of structurally resolved protein pairs, we generated a standard curve by plotting the $\mathrm{CBRET}$ ratios against the measured distances. We observed a strong negative correlation between the cBRET ratios and the reported distances $(R=-0.58)$, which is best described by a sigmoidal fit (Supplementary Figure $\mathbf{8 C}$ ). This is in good agreement with previous reports describing that the energy transfer efficiency is inversely proportional to the sixth power of the distance between the donor and the acceptor ${ }^{29}$. This result strongly suggests that cBRET ratios can indeed be used to measure distances between protein domains in live cells.

We next compared cBRET ratios measured between full-length proteins in cellulo, to 289 the reported distances in the corresponding high-resolution structures where truncated proteins were used. For example, in the structure of the DR1-DRAP1 PPI, all 176 amino acids 
of DR1 are resolved, while only the first 77 of the 205 amino acids of DRAP1 are structurally

292 defined ${ }^{33}$. Thus, $\sim 62 \%$ of the DRAP1 protein, including its entire C-terminal domain, does not 293 appear in the co-complex structure. The molecular distances reported in the structure suggest that the resolved termini of DRAP1 (N-terminus and glutamic acid 77) are both $\sim 20 \AA$ apart from the N-terminus of DR1 (Supplementary Figure 8D). When comparing these reported distances to the LuTHy-BRET results, we observed higher cBRET ratios when DRAP1 and DR1 were both $\mathrm{N}$-terminally tagged, compared to C-terminally fused DRAP1 and N-terminally fused DR1 (Supplementary Figure 8E). This suggests that the physical distance between the C-terminus of full-length DRAP1 and the N-terminus of DR1 is indeed larger in live cells than the $\sim 20 \AA$ reported in the structure where a truncated DRAP1 protein was used. To estimate the distance between the structurally unresolved termini in the full-length co-complex, we inferred the distance from the LuTHy-BRET data by using the calibrated cBRET-distance standard curve (Supplementary Figure $\mathbf{8 C}$ ). The measured cBRET ratios indicated that the DRAP1 C-terminus is $\sim 61 \AA$ apart from the N-terminus, and $\sim 74 \AA$ apart from the C-terminus of DR1 (Figure $4 \mathbf{H}, \mathrm{I})$. To generalize these findings, we extended the analysis to 30 additional PPIs that scored positive in LuTHy-BRET, and for which structural information on the tagged protein termini is currently missing (Figure $\mathbf{4 J}$ ). As expected, we found that distances between full-length proteins in live cells inferred from LuTHy-BRET ratios do not generally match with the structurally reported distances (Supplementary Figure 8F, Supplementary Figure 10), confirming the results obtained for the DR1-DRAP1 interaction.

Together, these findings confirm that LuTHy-BRET measurements can provide

312 valuable, complementary information about molecular distances between the subunits of protein complexes in live cells.

Assessing disease mutation-induced dynamics in structurally unresolved protein domains of the Huntingtin-HAP40 interaction mutation on the distance between protein domains, we studied the Huntington's disease 
related Huntingtin-HAP40 complex. Huntington's disease is caused by a CAG-repeat expansion located in exon-1 of the Huntingtin gene $(H T T)$ that is translated into an elongated polyglutamine (polyQ) stretch within the first 83 amino acids of the $>3000$ amino acids HTT protein $^{34,35}$. Recently, the structure of full-length HTT, in complex with its binding partner HAP40, was solved at high-resolution by cryo-EM (Figure 5A) $)^{36}$. However, the polyQ stretch located at the N-terminus of HTT was not structurally solved, probably due to its high flexibility ${ }^{36}$. Thus, the structure and relative localization of the $\mathrm{N}$-terminal, polyQ-containing domain remains elusive.

To investigate if the expansion mutation could influence the relative arrangement and molecular distance between the N-terminal, polyQ-containing domain of HTT and HAP40, we assessed this interaction by LuTHy-BRET. We observed a significant reduction in cBRET ratios when comparing the HTT-HAP40 interaction where HTT contains either a pathogenic (Q145), or a physiological (Q23) polyQ tract (Figure 5B). To determine the distance between the two proteins, we used the cBRET-distance standard curve (Supplementary Figure 8C) and found that the N-terminus of HAP40 is $~ 53 \AA$ apart from the N-terminus of HTT with the non-pathogenic Q23 tract (Figure 5A; Supplementary Table 7). However, the distance increases to $\sim 58 \AA$ when HTT contains the elongated, pathogenic Q145 stretch (Figure 5A; Supplementary Table 7). This suggests that the polyQ expansion mutation alters the domain arrangements within the HTT-HAP40 complex, and that LuTHy-BRET is a powerful tool to measure such subtle changes in live cells.

To assess if the effect of the elongated polyQ stretch on the relative arrangement of the N-terminal HTT domain is also detectable in the absence of HAP40, we analyzed the HTT protein in its apo form. The structure of the apo-HTT protein was recently solved and it was proposed that its C-terminal HEAT (Huntingtin/Elongation factor 3/protein phosphatase 2A/TOR) domain alters its position relative to the $\mathrm{N}$-terminal HEAT domain upon polyQ expansion ${ }^{37}$. To monitor such a dynamic structural change within the HTT protein in live cells, we generated an intramolecular LuTHy-BRET sensor, where the NanoLuc (NL) luciferase is

345 fused to the $\mathrm{N}$-terminus, and the fluorescent acceptor, mCitrine (mCit), is incorporated into an 
unstructured loop at leucine 2680 (L2680, Figure 5C-E). We then quantified LuTHy-BRET signals for the resulting NL-HTTQ23-mCit(L2680) and NL-HTTQ145-mCit(L2680) sensor proteins (Figure 5F). Using the cBRET-distance standard curve, we interpolated that the HTTQ23 N-terminus is $\sim 83 \AA$ apart from the loop at L2680, while this distance increases to 93 $\AA$ in the HTTQ145 protein (Figure 5D; Supplementary Table 7). Interestingly, it was also shown that the apo-HTT conformation significantly differs from the HTT-HAP40 complex ${ }^{37}$. Upon HAP40 binding, the C-terminal HEAT domain undergoes a rearrangement, swinging into closer proximity to the $\mathrm{N}$-terminus of HTT (Figure 5E). To monitor this dynamic rearrangement in live cells, we used the NL-HTTQ23-mCit(L2680) and NL-HTTQ145-mCit(L2680) LuTHy-BRET sensors and quantified the BRET ratios in presence or absence of exogenous HAP40 protein. When co-expressing HAP40 and the HTT sensors, the BRET ratio significantly increased, indicating that HTT also undergoes this structural rearrangement upon HAP40 binding in live cells (Figure 5F). In addition, in the presence of HAP40, the NL-HTTQ23-mCit(L2680) sensor showed a significantly higher BRET signal compared to the NL-HTTQ145-mCit(L2680) sensor, confirming that the polyQ expansion alters the domain arrangements of the HTT-HAP40 complex (Figure 5E). Using these data, we interpolated that in the presence of HAP40, the HTTQ23 N-terminus and the structural loop at L2680 are $\sim 61 \AA$ apart, whereas they are $\sim 71 \AA$ apart with the expanded polyQ tract (Figure 5D; Supplementary Table 7). Taken together, these results show that both in the presence or absence of HAP40, the pathogenic polyQ stretch-containing $\mathrm{N}$-terminal domain of HTT is extending $\sim 10 \AA$ further away from the core protein, a phenomenon that could not be resolved in the cryo-EM structures. Notably, this extension can make the $\mathrm{N}$-terminal region of the HTT mutant more accessible to engage in additional interactions, which is in good agreement with a previous report ${ }^{38}$.

Overall, these findings demonstrate that the LuTHy-BRET assay can be used to monitor dynamic changes and rearrangements (e.g. induced by mutations) within protein complexes in living cells. 


\section{DISCUSSION}

To globally understand cellular processes, it is essential to characterize complexomes to an extent where all interacting protein subunits of a complex and their relationships to one another are clearly defined. Currently, this knowledge is usually obtained when the 3D structure of a protein complex is resolved. In parallel, the $\mathrm{Y} 2 \mathrm{H}$ system has been widely used to generate large PPI networks based on relatively simple yes/no growth readouts. However, many binary PPI assays can generate highly quantitative scores. For example, such assays have been previously used to determine relative binding affinities or to assess the effects of mutations and drugs on specific PPIs ${ }^{19,39,40}$. Here, we demonstrate that the quantitative readouts of two versatile $\mathrm{PPI}$ assays, LuTHy and $\mathrm{mN} 2 \mathrm{H}$, can be used to systematically detect protein interactions within distinct multiprotein complexes, and confidently differentiate direct PPIs from indirect associations. The presented quantitative mapping approach can thus complement high-throughput AP-MS-based and low-throughput structural biology techniques to perform systematic, in-depth characterizations of diverse protein complexes.

We provide different standards to control for the quality of such quantitative mapping efforts: 1) the published hsPRS-v2 and hsRRS-v2, which contain direct interactions and random protein pairs, respectively ${ }^{18}$, and 2) the direct interactions and indirect associations from three multiprotein complexes, i.e. LAMTOR, BRISC, and MIS12. These pairs of fulllength proteins can systematically be introduced in future screening pipelines to calibrate PPI assays and experimentally determine scoring thresholds that will maximize detection of direct interactions, while minimizing recovery of indirect associations, inter-complex or random protein pairs. Interestingly, while LuTHy also identified indirect associations at a threshold where none of the inter-complex pairs were scored positive, the $\mathrm{mN} 2 \mathrm{H}$ assay did not recover any of those pairs. This could potentially be explained by the fact that LuTHy assays are performed under conditions of very low donor protein expression ${ }^{19}$. Therefore, it is likely that endogenously expressed members of the complex might bridge the indirectly associating proteins detected by LuTHy. However, direct interactions could confidently be distinguished 
401 from indirect associations using a machine learning-based clustering algorithm that should be

402 applicable to analyze the quantitative readouts of other binary PPI assays.

403 Our quantitative mapping approach should also help prioritizing high-confidence, direct

404 interactions for further hypothesis-driven experiments such as those where PPIs are used as

405 drug targets in chemical screens, or as candidates in mutagenesis studies. For example,

406 identifying directly interacting subunits within disease-relevant protein complexes can pave

407 the way for the development of small-molecule inhibitors or stabilizers of the studied complex.

408 In addition, when a direct interaction is identified, libraries of protein fragments ${ }^{41}$ could easily

409 be tested in order to identify minimal interacting domains between the two partners. This could,

410 for example, guide structural biology efforts to solve specific 3D co-complex structures.

411 Since many macromolecular assemblies still remain unresolvable by traditional

412 structural biology techniques, it is necessary to integrate complementary experimental data ${ }^{42}$

413 and theoretical approaches ${ }^{43-48}$. In addition, 3D structures often use truncated protein

414 constructs and provide snapshots of the studied complexes outside their cellular

415 environment ${ }^{28,43}$. Indeed, all direct interactions occurring in live cells might not always be

416 captured in the 3D model(s) currently available, and they are thus limited in fully representing

417 the in vivo dynamics of complexes with full-length proteins. However, data obtained by binary

418 interaction assays can provide important, complementary information on protein complex

419 architectures in a more physiological environment. For example, single-molecule Förster

420 resonance energy transfer (smFRET) has provided valuable results as an integrative

421 structural biology technique by delivering information on distances between protein subunits

422 within complexes in live cells ${ }^{28,31}$. We are now adding LuTHy-BRET to the list of available

423 integrative structural biology tools since we show that this readout can be used to measure

424 distances between protein subunits and domains as well as to monitor dynamic

425 conformational changes in live cells. Since LuTHy-BRET measurements are obtained from a population of molecules and not from a single molecule, it detects averaged effects within the studied cell population. Furthermore, BRET-based approaches offer three advantages over techniques like smFRET: 1) they can be applied in vivo ${ }^{49}$; 2) they have higher signal-to-noise 
ratios due to the absence of autofluorescence and photodestruction ${ }^{50}$; and 3) they do not

430 require specialized equipment for in-cell measurements and can thus be easily implemented

431 in any lab equipped with microtiter plate readers.

432 Finally, we have shown that the polyQ expansion mutation in the Huntington's disease related HTT protein gives rise to an abnormal domain rearrangement that can be quantified in

434 live cells with LuTHy-BRET. This suggests that this assay can potentially be used in future experiments to identify chemical modulators that reverse the effect of this and other mutations.

\section{AUTHOR CONTRIBUTIONS}

437 P.T., S.G.C., J.O., M.V. and E.E.W. conceived the study. P.T., C.S., S.G.C., J.O., P.C., Y.W., 438 E.S.R., S.G., M.Z., S.B., K.S. and Y.J. designed and performed the experiments and collected 439 the data. P.T. analyzed the majority of the results, with significant contributions from C.S., 440 S.G.C., J.O., M.S., T.H. while E.S.R., P.C., Y.W., J.C.T., M.A.C., D.E.H. and Y.J. provided 441 critical insights. All authors discussed the results and P.T., C.S., S.G.C., J.O., M.V. and E.E.W. 442 wrote the manuscript.

\section{ACKNOWLEDGEMENTS}

444 The authors would like to thank all members of the Wanker, Vidal, Jacob, and Twizere 445 laboratories for helpful discussions throughout this project. This work was supported by the 446 Helmholtz Association, iMed and Helmholtz-Israel Initiative on Personalized Medicine 447 (Germany); the Federal Ministry of Education and Research and e:med Systems Medicine IntegraMent 01GS0844 (Germany) all to E.E.W.; as well as by the CHDI Foundation (USA); 449 the German Cancer Consortium DKTK (Germany) and the Deutsche Krebshilfe, ENABLE

450 (Germany) to E.E.W. and P.T. This work was also supported by a Claudia Adams Barr Award 451 to S.G.C., a Fonds de la Recherche Scientifique (F.R.S.-FNRS)-Télévie Grant (FC27371, 452 Credit no 7454518F) and a Wallonia-Brussels International (WBI)-World Excellence 453 Fellowship to J.O. Additional supports were provided by NIH grants P50HG004233 and 
454 R01GM130885 awarded to M.V. and U41HG001715 awarded to M.V., D.E.H. and M.A.C. This

455 work was also supported by the LabEx IBEID (grant 10-LABX-0062). M.V. is a Chercheur

456 Qualifié Honoraire, and J.C.T. a Maître de Recherche of the Fonds de la Recherche

457 Scientifique (F.R.S.-FNRS, Wallonia-Brussels Federation, Belgium).

\section{CONFLICT OF INTEREST}

459 The authors declare that they have no conflict of interest.

\section{DATA AND CODE AVAILABILITY}

461 The protein interactions from this publication have been submitted to the IMEx 462 (http://www.imexconsortium.org) consortium through IntAct ${ }^{51}$ and assigned the identifier 463 IM-29174. All UniProt and RCSB-PDB accession codes are provided in the Source Data. 464 Python and $\mathrm{R}$ codes used for data analyses are available upon request.

\section{METHODS}

\section{ORF sequencing and plasmid generation}

467 For hsPRS-v2 and hsRRS-v2 proteins, the corresponding sequence-verified entry vectors 468 published in Choi et al ${ }^{18}$ were Gateway cloned into the different LuTHy destination plasmids. ORFs for subunits of the LAMTOR, MIS12 and BRISC complexes were taken from the CCSB

470 human ORFeome 8.1, which is a sequence confirmed clonal collection of human ORFs in a 471 Gateway entry vector system ${ }^{24}$. In total, 16 entry plasmids were picked from the collection, 472 single clones were isolated, and ORFs were PCR-amplified and confirmed by bi-directional 473 Sanger DNA sequencing. Entry clones were shuttled into LuTHy and N2H destination vectors 474 using the Gateway Cloning Technology. All resulting vectors were analyzed by PCRamplification of cloned ORFs and DNA gel electrophoresis (N2H plasmids), or restriction digestion and sequence validation (LuTHy plasmids). For the LuTHy assay, additional control 
477 plasmids (PA-NL, Addgene \#113445; PA-mCit-NL, Addgene \#113444; PA-mCit, Addgene 478 \#113443; NL, Addgene \#113442) were used, as previously described ${ }^{16}$. The pcDNA3.1 479 plasmids encoding HTTQ23 and HTTQ145 (glutamines encoded by CAG/CAA triplets) were 480 gifts from the $\mathrm{CHDI}$ foundation that were subcloned into pDONR221 entry vectors 481 (ThermoFisher, \#12536017). The pDONR221-F8A1 entry plasmid encoding HAP40 was obtained from Source BioSciences (OCAAo5051D1091D). HTT and HAP40 entry clones were shuttled into LuTHy destination plasmids, and HAP40 was also cloned into a pDEST26-cmyc destination plasmid, which was a kind gift from Matthias Selbach. All amino acid positions in the HTT protein refer to a sequence containing 17 glutamines with a total length of 3138 amino acids. To generate the intramolecular LuTHy-BRET HTT sensors, the LuTHy plasmids pcDNA3.1 NL-HTTQ23 and pcDNA3.1 NL-HTTQ145 were digested with Sfil and Sfol that cut within the HTT sequence and the Kan/neoR resistance sequence of the plasmid backbone. The resulting larger fragment (12161 bp for Q23, and $12563 \mathrm{bp}$ for Q145) were agarose gel purified using the Invisorb ${ }^{\circledR}$ Fragment Cleanup kit from Invitek Molecular. Next, the HTT sequence from Q2500 to L2680 was PCR amplified using the primers 353-FWD and 341REV. The 353-FWD primer contained a 30 bp overhang into the HTT sequence, and the 341REV primer an 18 bp GSGSGS-linker sequence as well as a 7 bp overhang into the 5'mCitrine sequence. The coding sequence for $\mathrm{mCitrine}$ was amplified without the start and stop codons, using the 342-FWD and the 343-REV primers. The 342-FWD and 343-REV primers both contained an 18 bp GSGSGS-linker sequence and a 7 bp overhang into the respective 5' or 3' HTT sequences. The HTT sequence from amino acid P2681 to the Kan/neoR cassette of the plasmid backbone was amplified using the following primers: 340-FWD and 354-REV. The 340-FWD primer contained an 18 bp GSGSGS-linker sequence and a 7 bp overhang into the 3'-mCitrine sequence, and the 354-REV primer a 30 bp overhang into the Kan/neoR cassette of the plasmid backbone. All PCR products were column purified using the $\mathrm{MSB}^{\circledR}$ Spin PCRapace kit from Invitek Molecular. Finally, the digested and gel purified plasmid backbones were assembled, together with the three purified PCR products, by Gibson assembly according to the manufacturer's protocol (New England Biolabs, E2611). The 
505 resulting colonies carrying the final plasmids were analyzed by restriction digestion and

506 Sanger DNA sequencing. All primer sequences can be found in Supplementary Table 8.

\section{LuTHy assay procedure}

508 The LuTHy assay was performed as previously described ${ }^{19}$. In brief, HEK293 cells were reversely transfected in white 96-well microtiter plates (Greiner, \#655983) at a density of 4.0-

$5104.5 \times 10^{4}$ cells per well with plasmids encoding donor and acceptor proteins. After incubation

511 for $48 \mathrm{~h}$, mCitrine fluorescence was measured in intact cells (Ex/Em: $500 \mathrm{~nm} / 530 \mathrm{~nm}$ ). For

512 LuTHy-BRET assays, coelenterazine-h (pjk, \#102182) was added to a final concentration of $5135 \mu \mathrm{M}(5 \mathrm{mM}$ stock dissolved in methanol). Next, cells were incubated for an additional $15 \mathrm{~min}$ 514 and total luminescence as well as luminescences at short (370-480 nm) and long (520-570 $515 \mathrm{~nm}$ ) wavelengths were measured using the Infinite ${ }^{\circledR}$ microplate readers $M 200, M 1000$, or 516 M1000 PRO (Tecan). After luminescence measurements, the luminescence-based co517 precipitation (LuC) assay was performed. Cells were lysed in 50-100 $\mu$ L HEPES-phospho518 lysis buffer (50 mM HEPES, $150 \mathrm{mM} \mathrm{NaCl}, 10 \%$ glycerol, 1\% NP-40, 0.5\% deoxycholate, 20 $519 \mathrm{mM} \mathrm{NaF}, 1.5 \mathrm{mM} \mathrm{MgCl}_{2}, 1 \mathrm{mM}$ EDTA, $1 \mathrm{mM} \mathrm{DTT}, 1 \mathrm{U}$ Benzonase, protease inhibitor cocktail 520 (Roche, EDTA-free), 1 mM PMSF, 25 mM glycerol-2-phosphate, 1 mM sodium orthovanadate, $2 \mathrm{mM}$ sodium pyrophosphate) for $30 \mathrm{~min}$ at $4^{\circ} \mathrm{C}$. Lysates $(7.5 \mu \mathrm{L})$ were transferred into small volume 384-well microtiter plates (Greiner, \#784074) and fluorescence (mCit $\left.\mathrm{I}_{\mathrm{N}}\right)$ was measured as previously described ${ }^{19}$. To measure the total luminescence $\left(\mathrm{NL}_{\mathrm{IN}}\right), 7.5 \mu \mathrm{L}$ of $20 \mu \mathrm{M}$ coelenterazine-h in PBS was added to each well and the plates incubated for 15 more minutes. For LuC, small volume 384-well microtiter plates (Greiner, \#784074) were coated with sheep gamma globulin (Jackson ImmunoResearch, \#013-000-002) in carbonate buffer (70 mM $\mathrm{NaHCO}_{3}, 30 \mathrm{mM} \mathrm{Na} \mathrm{CO}_{3}, \mathrm{pH}$ 9.6) for $3 \mathrm{~h}$ at room temperature, and blocked with $1 \% \mathrm{BSA}$ in carbonate buffer before being incubated overnight at $4^{\circ} \mathrm{C}$ with rabbit anti-sheep lgGs in carbonate buffer (Jackson ImmunoResearch, \#313-005-003). $15 \mu \mathrm{L}$ of cell lysate was

530 incubated for $3 \mathrm{~h}$ at $4^{\circ} \mathrm{C}$ in the IgG-coated 384-well plates. Then, all wells were washed three 531 times with lysis buffer and mCitrine fluorescence (mCitout) was measured as described ${ }^{19}$. 
532 Finally, $15 \mu \mathrm{L}$ of PBS buffer containing $10 \mu \mathrm{M}$ coelenterazine-h was added to each well and

533 luminescence (NLOUT) was measured after a 15 min incubation period.

\section{LuTHy data analysis}

535 Data analysis was performed as previously described ${ }^{19}$. In brief, the LuTHy-BRET and LuTHy-

536 LuC ratios from BRET and co-precipitation measurements are calculated as follows:

(1) BRET ratio $=\frac{L W L}{S W L}-C f$ with $C f=\frac{L W L_{P A-N L}}{S W L_{P A-N L}}$

538 with LWL and SWL being the detected luminescences at long (520-570 nm) and short (370-

$539480 \mathrm{~nm}$ ) wavelengths, respectively. The correction factor (Cf) represents the donor bleed-

540 through value from the PA-NL only construct. The corrected BRET (cBRET) ratio is calculated

541 by subtracting the maximum BRET ratios of control 1 (NL/PA-mCit-Y), or of control 2 (NL-

542 X/PA-mCit) from the BRET ratio of the studied interaction (NL-X/PA-mCit-Y).

543 For the LuC readout, the obtained luminescence precipitation ratio (PIR) of the control protein

544 PA-NL (PIR PA-NL ) is used for data normalization, and is calculated as follows:

545

(2) $P I R_{P A-N L}=\frac{N L_{O U T}}{2 * N L_{I N}}$

546 With NLout being the total luminescence measured after co-IP and NLIN the luminescence

547 measured in the cell extracts, directly after lysis. Subsequently, LuC ratios are calculated for

548 all interactions of interest, and normalized to the PIR $\mathrm{PA}-\mathrm{NL}_{\mathrm{L}}$ ratio:

549

(3) LuC ratio $=\frac{N L_{O U T} / 2 * N L_{I N}}{P I R_{P A-N L}}$

550 Finally, a corrected LuC (cLuC) ratio is calculated by subtracting either the LuC ratio of 551 control 1 (NL/PA-mCit-Y), or of control 2 (NL-X/PA-mCit) from the LuC ratio of the studied 552 interaction (NL-X/PA-mCit-Y). The calculated LuC ratios obtained for controls 1 and 2 are then 553 compared to each other, and the highest value is used to correct the LuC ratio of the respective 554 interaction. 
556 HEK293T cells were seeded at $6 \times 10^{4}$ cells per well in 96-well, flat-bottom, cell culture 557 microplates (Greiner Bio-One, \#655083), and cultured in Dulbecco's modified Eagle's medium 558 (DMEM) supplemented with $10 \%$ fetal calf serum at $37^{\circ} \mathrm{C}$ and $5 \% \mathrm{CO}_{2} .24 \mathrm{~h}$ later, cells were transfected with $100 \mathrm{ng}$ of each $\mathrm{N} 2 \mathrm{H}$ plasmid (pDEST-N2H-N1, -N2, -C1 or -C2) using linear polyethylenimine (PEI) to co-express proteins fused with complementary NanoLuc fragments, was prepared according to the manufacturer's instructions. Briefly, $200 \mathrm{mg}$ of $\mathrm{PEI} \mathrm{HCl}$ powder were added to $170 \mathrm{~mL}$ of water, stirred until complete dissolution, and $\mathrm{pH}$ was adjusted to 7 with $1 \mathrm{M} \mathrm{NaOH}$. Water was added to obtain a final concentration of $1 \mathrm{mg} / \mathrm{mL}$, and the stock solution was filtered through a $0.22 \mu \mathrm{m}$ membrane. The DNA/PEI ratio used for transfection was 1:3 (mass:mass). $24 \mathrm{~h}$ after transfection, the culture medium was removed and $50 \mu \mathrm{L}$ of 100x diluted NanoLuc substrate (Furimazine, Promega Nano-Glo, N1120) was added to each well of a 96-well microplate containing the transfected cells. Plates were incubated for $3 \mathrm{~min}$ at room temperature. Luciferase enzymatic activity was measured using a TriStar or CentroXS

570 luminometer (Berthold; $2 \mathrm{~s}$ integration time).

\section{$571 \quad$ Processing publicly available interaction data}

572 Publicly available binary protein interaction datasets used in this study came from the original

573 Choi et al publication ${ }^{18}$. The hsPRS-v2 PPI recovery rates were extracted as published, in 574 conditions where none of the hsRRS-v2 pairs are scored positive. To generate hsPRS-PDB, 575 structurally supported interactions from hsPRS-v2 were identified using interactome insider 576 (http://interactomeinsider.yulab.org) ${ }^{52}$, and using both, co-crystal structures and homology 577 modeling (Source Data Figures 1-2). The BioPlex dataset ${ }^{7,11,14}$ was downloaded from 578 https://bioplex.hms.harvard.edu/interactions.php on May 10, 2021. 
579 Scoring hsPRS-v2 PPls under conditions where no hsRRS-v2 pair is scored positive

580 for LuTHy

581 Source data for each assay version performed in this study are presented in the Source Data

582 Figures 1-2. Each binary PPI experiment with LuTHy-BRET and LuTHy-LuC was performed six times, with two biological and three technical replicates. For each protein pair X-Y, we calculated the corrected BRET and LuC ratios as described above. To identify protein pairs that scored positive at a threshold of no hsRRS-v2 detection for a given tagging configuration, only cBRET or cLuC ratios higher than the highest hsRRS-v2 score for that tagging configuration were considered. All hsPRS-v2 pairs that did not meet this criterion were not scored positive and therefore defined as not detected in the corresponding assay version.

\section{Complex selection and definition of direct interactions and indirect associations}

590 Human protein complexes used in this study were selected based on the following criteria.

591 First, human protein complexes should have at least one experimentally determined structure in $\mathrm{PDB}^{23}$ (https://www.rcsb.org). Second, the complex should have at least four subunits. Third, at least $80 \%$ of entry clones for individual subunits of a complex should be present in the human ORFeome 8.1 collection $^{24}$. A total of 24 distinct complexes (Supplementary Table 1) with different PDB structures met those criteria, and three protein complexes with well-documented biological functions were selected from this list: LAMTOR, BRISC and MIS12. These complexes were also picked as they appear in AP-MS interactome maps, according to BioPlex ${ }^{7,11,14}$. Finally, direct interactions and indirect associations within protein complexes were determined by interactome $3 D^{25}$ and PDBePISA ${ }^{26}$.

\section{Scoring interactions within multiprotein complexes}

601 Data for LuTHy and mN2H mapping of multiprotein complexes can be found in Source Data

602 Figures 3-5. Recovery of interactions by LuTHy-BRET, LuTHy-LuC and mN2H was 603 calculated based on construct-specific cutoffs. Therefore, the quantitative scores were 
604 determined as described in the LuTHy and $\mathrm{mN} 2 \mathrm{H}$ methods sections, respectively. For each

605 construct, $X$ or $Y$, involved in the $X-Y$ interaction, a cutoff was defined as the highest inter606 complex pair score for each of the two respective constructs ( $\mathrm{X}$ and $\mathrm{Y})$. The final cutoff for a 607 tested protein pair between constructs $X$ and $Y$ was then determined as the maximum of the 608 two cutoff values.

\section{Cluster analysis of LuTHy data}

610 For supervised classification of direct and not-direct interaction clusters, a Gaussian finite 611 mixture model using the R package mclust $^{53}$ was used. First, the LuTHy-BRET and LuTHy612 LuC quantitative scores (i.e. cBRET and CLuC ratios) from 63 LuTHy-positive interactions among hsPRS-PDB and from 585 protein pairs in hsRRS-v2 (Supplementary Table 3) were used as positive and negative training sets, respectively. The MclustDA discriminant analysis

615 function was applied to those training sets using the eigenvalue decomposition discriminant 616 analysis (EDDA) method. The training set performed at a $2.36 \%$ classification error rate. Next, 617 the trained algorithm was used to cluster 117 intra-complex protein pairs (Supplementary

618 Table 3) that scored above the construct-specific cutoffs in LuTHy assays. Using the function 619 predict.MclustDA, 47 direct protein pairs (i.e. multiple configurations corresponding to 14 out of the 23 LuTHy-positive direct interactions) were correctly classified as direct interactions and all 12 indirect protein pairs (i.e. multiple configuration corresponding to all 6 LuTHy-positive

622 indirect associations) were accurately classified as not-direct. In addition, 58 direct protein 623 pairs (i.e. multiple configuration corresponding to 9 out of the 23 LuTHy-positive direct 624 interactions) could not be classified as direct interactions using this supervised classification 625 analysis.

626 Determining interaction interface areas, complex surface areas and molecular 627 distances between protein termini

628 Interaction interface and total complex surface areas were determined according to 629 PDBePISA ${ }^{26}$ (https://www.ebi.ac.uk/msd-srv/prot_int/pistart.html) on April 16, 2021. Protean 
630 3DTM (DNASTAR ${ }^{\circledR}$ ) was used to measure molecular distances between protein termini of

631 interactions in the hsPRS-PDB as well as between direct interactions and indirect associations

632 within the multiprotein complexes: LAMTOR (RCSB PDB: 6EHR), MIS12 (RCSB PDB: 5LSJ)

633 and BRISC (RCSB PDB: 6H3C). The determined molecular distances can be found in Source

634 Data Figures 3-5. Nonlinear and linear regressions between cBRET ratios and molecular 635 distances between protein termini were calculated using GraphPad Prism 9 and the fits were 636 compared using the extra-sum-of-squares $\mathrm{F}$ test with a bottom constraint to 0 . GraphPad

637 Prism 9 was also used to interpolate molecular distances and their $90 \%$ confidence intervals 638 using the measured cBRET ratios.

639

640 
1. Vidal, M., Cusick, M. E. \& Barabási, A.-L. Interactome networks and human disease. Cell 144, 986998 (2011).

2. Gorka, M. et al. Protein Complex Identification and quantitative complexome by $\mathrm{CN}$ PAGE. Sci Rep-uk 9, 11523 (2019).

3. Anger, A. M. et al. Structures of the human and Drosophila $80 \mathrm{~S}$ ribosome. Nature 497 , 80-85 (2013).

4. Natchiar, S. K., Myasnikov, A. G., Kratzat, H., Hazemann, I. \& Klaholz, B. P. Visualization of chemical modifications in the human $80 S$ ribosome structure. Nature 551, 472-477 (2017).

5. Lu, Y. et al. Conformational Landscape of the p28-Bound Human Proteasome Regulatory Particle. Mol Cell 67, 322-333.e6 (2017).

6. Hein, M. Y. et al. A human interactome in three quantitative dimensions organized by stoichiometries and abundances. Cell 163, 712723 (2015).

7. Huttlin, E. L. et al. The BioPlex Network: A Systematic Exploration of the Human Interactome. Cell 162, 425440 (2015).

8. Luck, K., Sheynkman, G. M., Zhang, I. \& Vidal, M. Proteome-Scale Human Interactomics. Trends Biochem Sci 42, 342-354 (2017).

9. Roux, K. J., Kim, D. I., Raida, M. \& Burke, B. A promiscuous biotin ligase fusion protein

661 identifies proximal and interacting proteins in mammalian cells. J Cell Biology 196, 801810 (2012).

10. Luck, K. et al. A reference map of the human binary protein interactome. Nature $\mathbf{5 8 0}$, 402-408 (2020).

11. Huttlin, E. L. et al. Dual proteome-scale networks reveal cell-specific remodeling of the human interactome. Cell 184, 3022-3040.e28 (2021).

12. Drew, K. et al. Integration of over 9,000 mass spectrometry experiments builds a global map of human protein complexes. Mol Syst Biol 13, 932 (2017).

669

670

671

672

673

674

675
13. Drew, K., Wallingford, J. B. \& Marcotte, E. M. hu.MAP 2.0: integration of over 15,000 proteomic experiments builds a global compendium of human multiprotein assemblies. Mol Syst Biol 17, (2021).

14. Huttlin, E. L. et al. Architecture of the human interactome defines protein communities and disease networks. Nature 545, 505 (2017).

15. Rolland, T. et al. A proteome-scale map of the human interactome network. Cell 159, 12121226 (2014). 
16. Yu, H. et al. High-Quality Binary Protein Interaction Map of the Yeast Interactome Network. Science 322, 104-110 (2008).

17. Venkatesan, K. et al. An empirical framework for binary interactome mapping. Nat Methods 6, 8390 (2009).

18. Choi, S. G. et al. Maximizing binary interactome mapping with a minimal number of assays. Nat Commun 10, 3907 (2019).

19. Trepte, P. et al. LuTHy: a double-readout bioluminescence-based two-hybrid technology for quantitative mapping of protein-protein interactions in mammalian cells. Mol Syst Biol 14, e8071 (2018).

20. Araujo, M. E. G. de et al. Crystal structure of the human lysosomal mTORC1 scaffold complex and its impact on signaling. Science 358, 377-381 (2017).

21. Petrovic, A. et al. Structure of the MIS12 Complex and Molecular Basis of Its Interaction with CENP-C at Human Kinetochores. Cell 167, 1028-1040.e15 (2016).

22. Rabl, J. et al. Structural Basis of BRCC36 Function in DNA Repair and Immune Regulation. Mol Cell 75, 483-497.e9 (2019).

23. Berman, H. M. et al. The Protein Data Bank. Nucleic Acids Res 28, 235-242 (2000).

24. Yang, X. et al. A public genome-scale lentiviral expression library of human ORFs. Nat Methods 8, 659661 (2011).

25. Mosca, R., Céol, A. \& Aloy, P. Interactome3D: adding structural details to protein networks. Nat Methods 10, 4753 (2012).

26. Krissinel, E. \& Henrick, K. Inference of Macromolecular Assemblies from Crystalline State. J Mol Biol 372, 774-797 (2007).

27. Chen, J., Sawyer, N. \& Regan, L. Protein-protein interactions: General trends in the relationship between binding affinity and interfacial buried surface area. Protein Sci $\mathbf{2 2}$, 510-515 (2013).

28. Lerner, E. et al. Toward dynamic structural biology: Two decades of single-molecule Förster resonance energy transfer. Science 359, eaan1133 (2018).

29. Stryer, L. \& Haugland, R. P. Energy transfer: a spectroscopic ruler. Proc National Acad Sci 58, 719-726 (1967).

30. Stryer, L. Fluorescence energy transfer as a spectroscopic ruler. Annu Rev Biochem 47, 819846 (1978).

31. Asher, W. B. et al. Single-molecule FRET imaging of GPCR dimers in living cells. Nat Methods 1-9 (2021) doi:10.1038/s41592-021-01081-y.

32. Quast, R. B. \& Margeat, E. Single-molecule FRET on its way to structural biology in live cells. Nat Methods 1-2 (2021) doi:10.1038/s41592-021-01084-9. 
33. Kamada, K. et al. Crystal Structure of Negative Cofactor 2 Recognizing the TBP-DNA Transcription Complex. Cell 106, 71-81 (2001).

34. MacDonald, M. E. et al. A novel gene containing a trinucleotide repeat that is expanded and unstable on Huntington's disease chromosomes. Cell 72, 971-983 (1993).

35. Scherzinger, E. et al. Huntingtin-Encoded Polyglutamine Expansions Form Amyloid-like Protein Aggregates In Vitro and In Vivo. Cell 90, 549-558 (1997).

36. Guo, Q. et al. The cryo-electron microscopy structure of huntingtin. Nature 555, 117120 (2018).

37. Jung, T. et al. The Polyglutamine Expansion at the N-Terminal of Huntingtin Protein Modulates the Dynamic Configuration and Phosphorylation of the C-Terminal HEAT Domain. Structure 28, 1035-1050.e8 (2020).

38. Harding, R. J. et al. Design and characterization of mutant and wild-type huntingtin proteins produced from a toolkit of scalable eukaryotic expression systems. J Biol Chem jbc.RA118.007204 (2019) doi:10.1074/jbc.ra118.007204.

39. Trepte, P. et al. DULIP: A Dual Luminescence-Based Co-Immunoprecipitation Assay for Interactome Mapping in Mammalian Cells. J Mol Biol 427, 33753388 (2015).

40. Saraon, P. et al. A drug discovery platform to identify compounds that inhibit EGFR triple mutants. Nat Chem Biol 1-10 (2020) doi:10.1038/s41589-020-0484-2.

41. Boxem, M. et al. A protein domain-based interactome network for C. elegans early embryogenesis. Cell 134, 534545 (2008).

42. Braberg, H. et al. Genetic interaction mapping informs integrative structure determination of protein complexes. Science 370, eaaz4910 (2020).

43. Ward, A. B., Sali, A. \& Wilson, I. A. Integrative Structural Biology. Science 339, 913-915 (2013).

44. Rout, M. P. \& Sali, A. Principles for Integrative Structural Biology Studies. Cell 177, 1384-1403 (2019).

45. Senior, A. W. et al. Improved protein structure prediction using potentials from deep learning. Nature 577, 706-710 (2020).

46. Baek, M. et al. Accurate prediction of protein structures and interactions using a threetrack neural network. Science eabj8754 (2021) doi:10.1126/science.abj8754.

47. Tunyasuvunakool, K. et al. Highly accurate protein structure prediction for the human proteome. Nature 1-9 (2021) doi:10.1038/s41586-021-03828-1.

48. Jumper, J. et al. Highly accurate protein structure prediction with AlphaFold. Nature 1-11 (2021) doi:10.1038/s41586-021-03819-2.

49. Mezzanotte, L., Root, M. van 't, Karatas, H., Goun, E. A. \& Löwik, C. W. G. M. In Vivo Molecular Bioluminescence Imaging: New Tools and Applications. Trends Biotechnol 35, 640652 (2017). 
50. Boute, N., Jockers, R. \& Issad, T. The use of resonance energy transfer in highthroughput screening: BRET versus FRET. Trends Pharmacol Sci 23, 351-354 (2002).

51. Orchard, S. et al. The MIntAct project-IntAct as a common curation platform for 11 molecular interaction databases. Nucleic Acids Res 42, D358-D363 (2014).

52. Meyer, M. J. et al. Interactome INSIDER: a structural interactome browser for genomic studies. Nature Methods 15, 107-114 (2018).

53. Scrucca, L., Fop, M., Murphy, T. B. \& Raftery, A. E. mclust 5: Clustering, Classification and Density Estimation Using Gaussian Finite Mixture Models. R J 8, 289-317 (2016). 
FIGURES
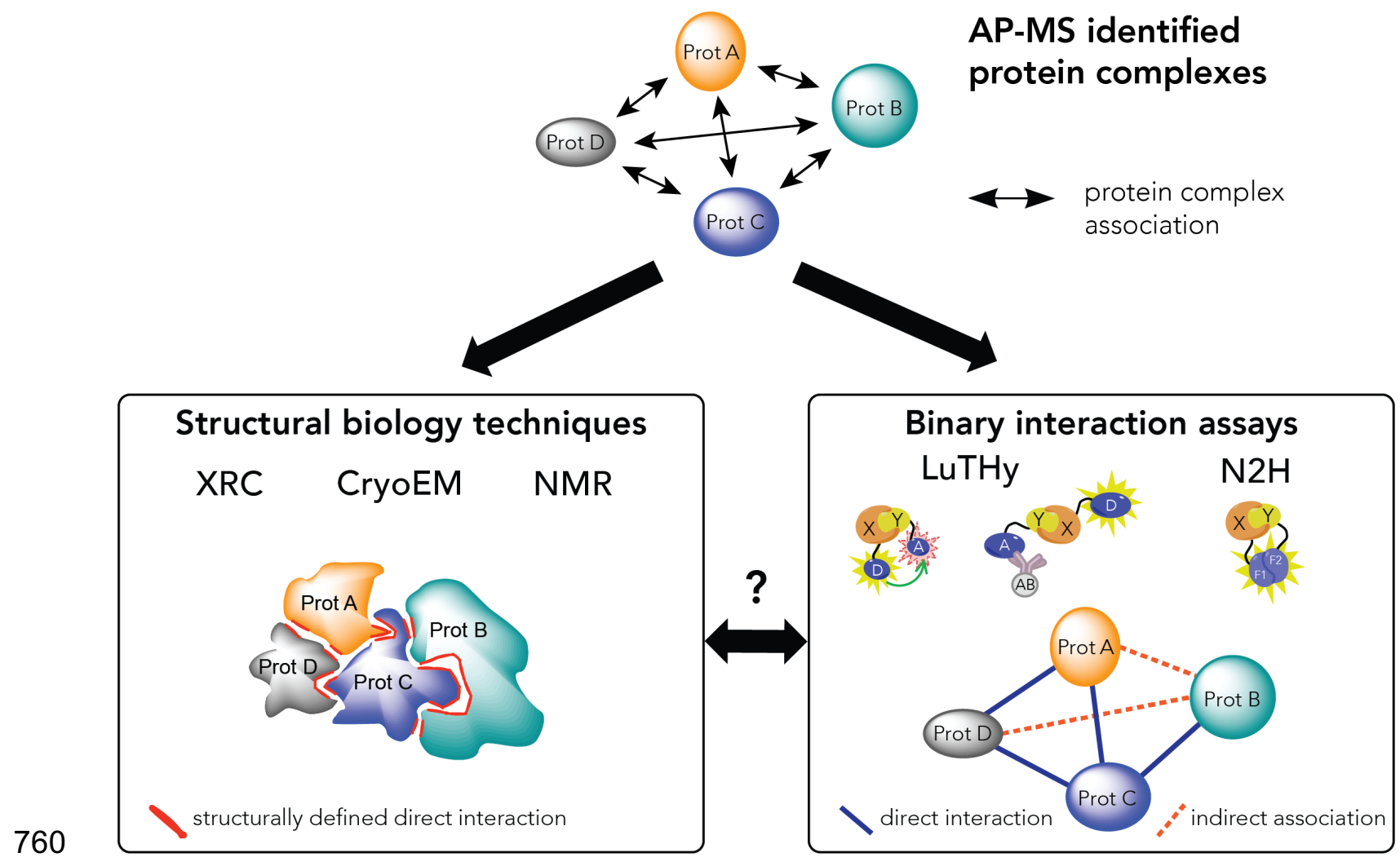
A

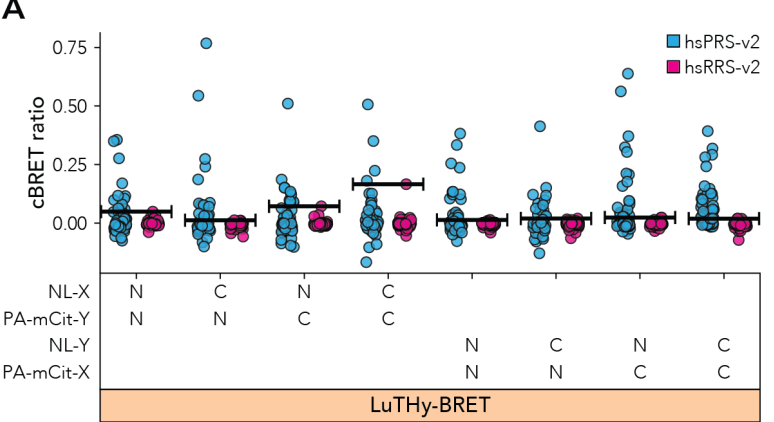

B

C
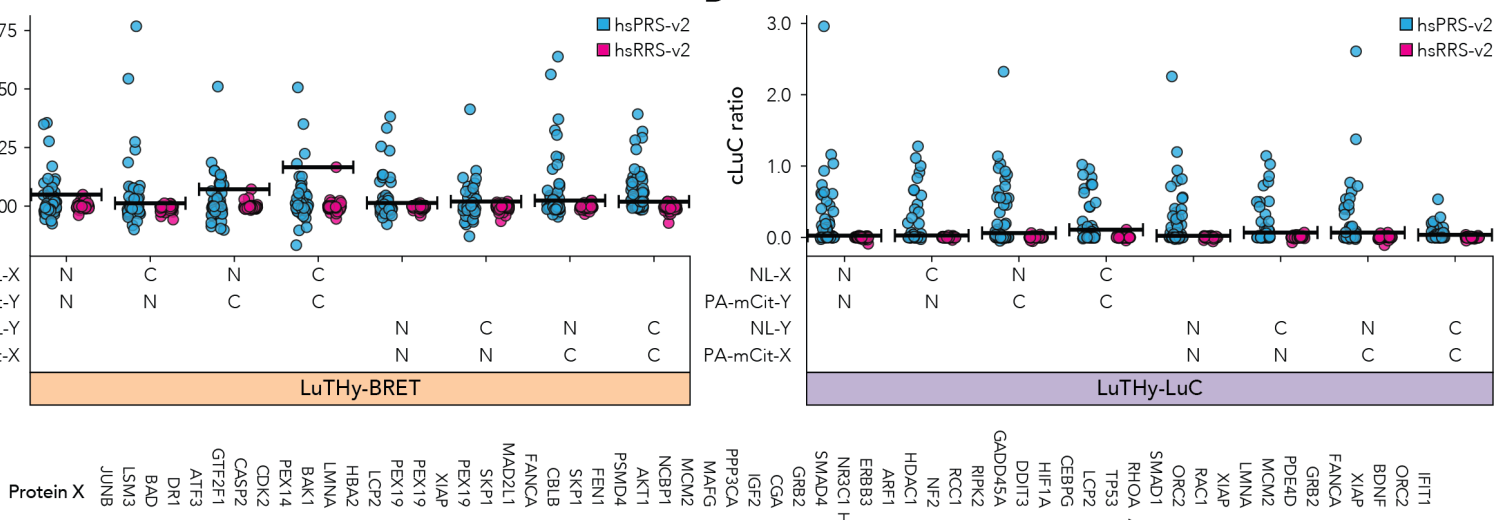

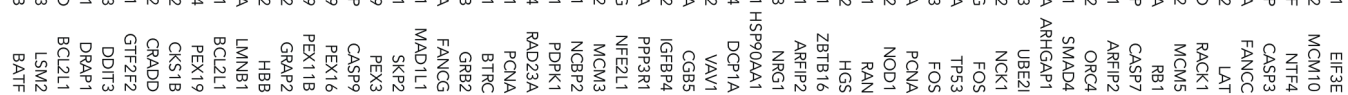

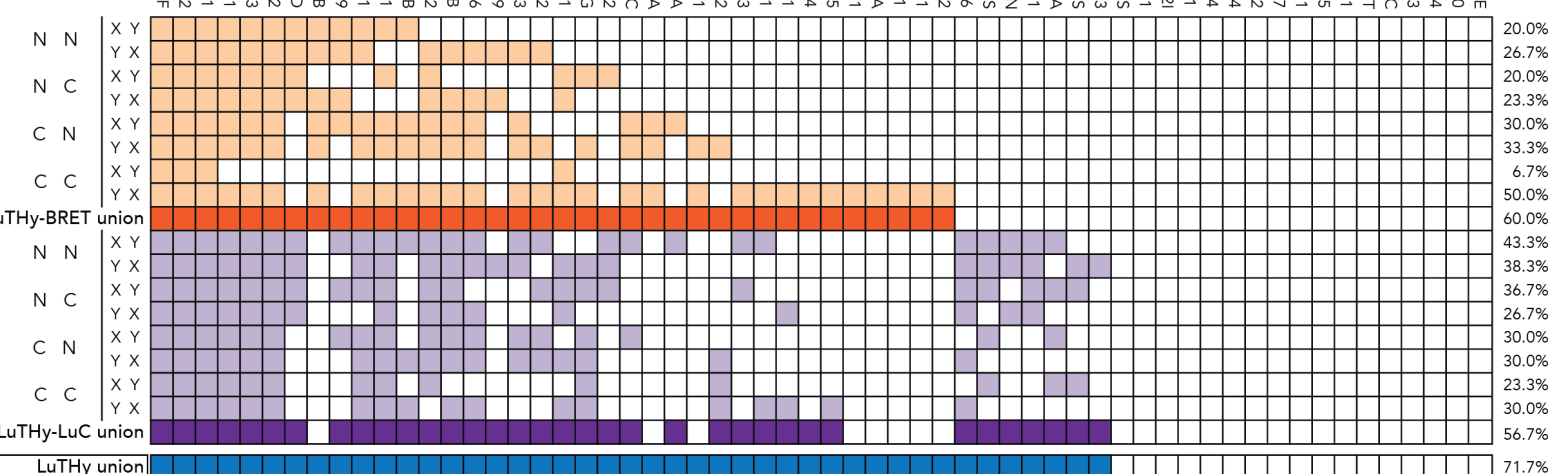

$D_{\text {वें }}$

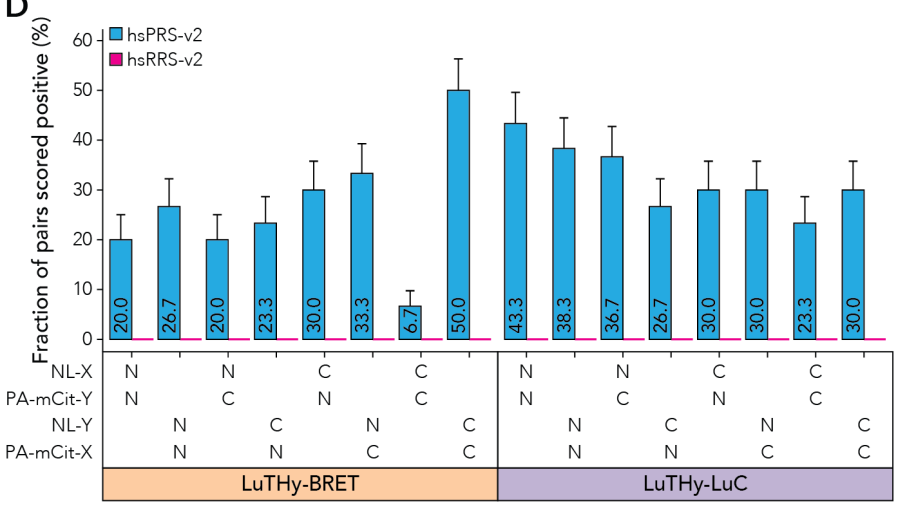

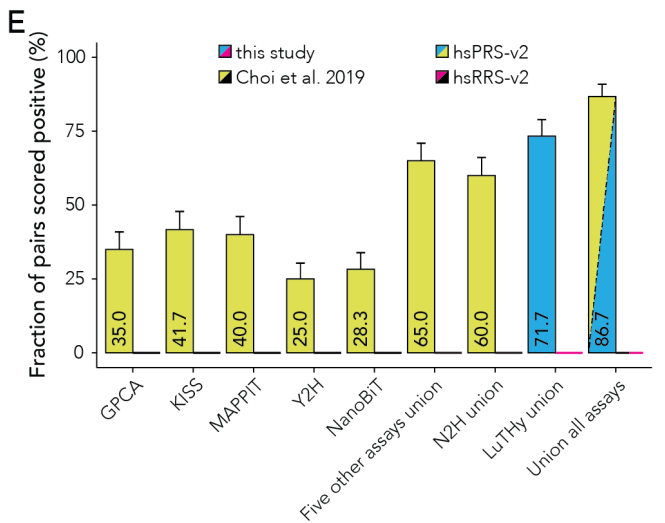

765 Quantitative scores for hsPRS-v2 and hsRRS-v2 pairs differentiated by tagging configurations for (A) LuTHy-BRET and (B) LuTHy-LuC. Horizontal lines in (A) and (B) indicate the scoring cutoffs for each configuration above which no hsRRS-v2 pair is scored positive. (C) Overview of the positively scored interactions from hsPRS-v2. Positive LuTHy-BRET interactions in different orientations (light orange) and their union (dark orange) are shown, as well as positive LuTHy-LuC interactions in the eight different configurations (light purple) and their union (dark purple). LuTHy union (blue) summarizes results for all 16 versions. The percentage (\%) at the end of each row represents the fraction of hsPRS-v2 PPIs scored positive. (D) Recovery rates of hsPRS-v2 interactions at no hsRRS-v2 
bioRxiv preprint doi: https://doi.org/10.1101/2021.08.25.457734; this version posted August 26,2021 . The copyright holder for this preprint

(which was not certified by peer review) is the author/funder, who has granted bioRxiv a license to display the preprint in perpetuity. It is made available under aCC-BY-NC-ND 4.0 International license.

772 detection for the eight different tagging configurations in LuTHy-BRET and LuTHy-LuC. (E) Comparison of hsPRS-

773 v2 recovery rates for LuTHy (LuTHy union) to other binary interaction methods benchmarked in Choi et al ${ }^{18}$. Error

774 bars indicate standard errors of the proportion in (D) and (E). 
A

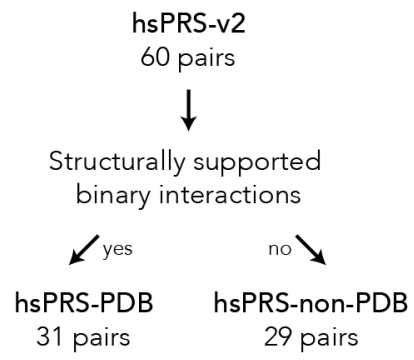

C

Figure 2. Benchmarking binary PPI assays against structurally supported interactions in hsPRS-v2.

777 (A) hsPRS-v2 contains 60 binary PPIs among which 31 pairs are supported by structural data in PDB (hsPRS-

778 PDB). The 29 remaining pairs are not currently supported by 3D structures (hsPRS-non-PDB). (B,C) Comparison

779 of hsPRS-PDB and hsPRS-non-PDB recovery rates from different binary interaction methods when none of the

780 hsPRS-v2 pairs are scored positive. Binary PPI assays combined ("Union all assays") recover all but one of the 781 hsPRS-PDB interactions. The percentage (\%) at the end of each row in (C) indicates the fraction of hsPRS-v2 PPIs
B

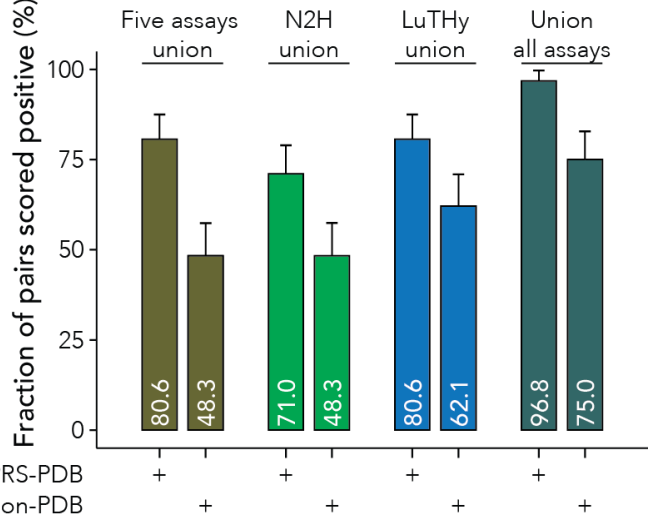

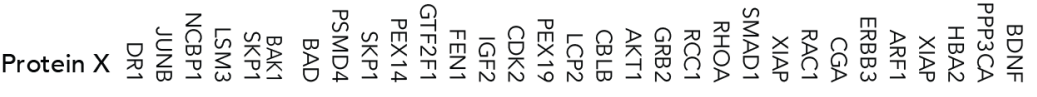

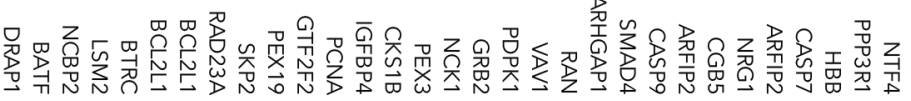

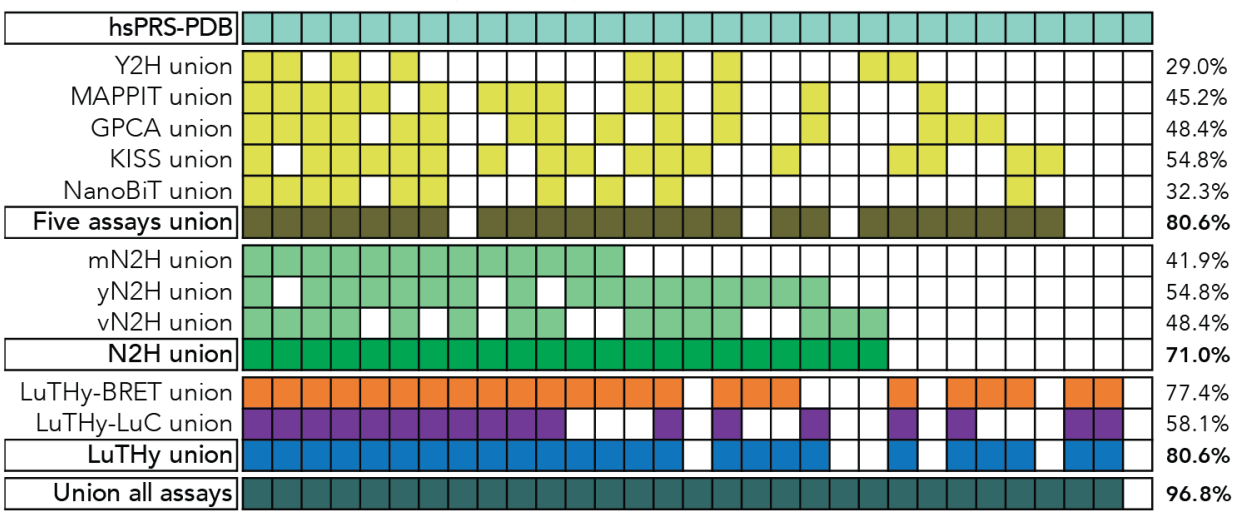
scored positive at no hsRRS-v2 detection. Error bars in (B) indicate standard errors of the proportion. 
bioRxiv preprint doi: https://doi.org/10.1101/2021.08.25.457734; this version posted August 26, 2021. The copyright holder for this preprint

(which was not certified by peer review) is the author/funder, who has granted bioRxiv a license to display the preprint in perpetuity. It is made available under aCC-BY-NC-ND 4.0 International license.

A

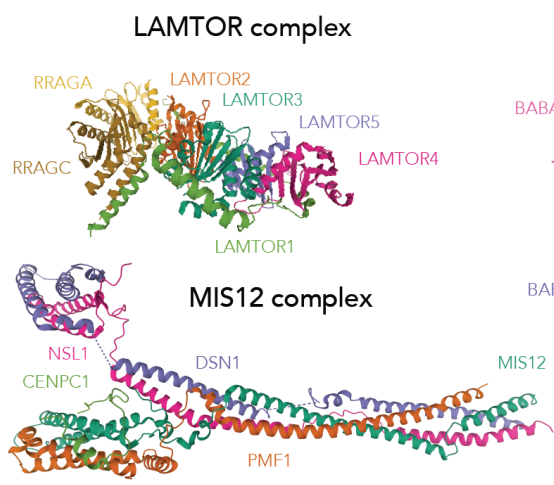

B

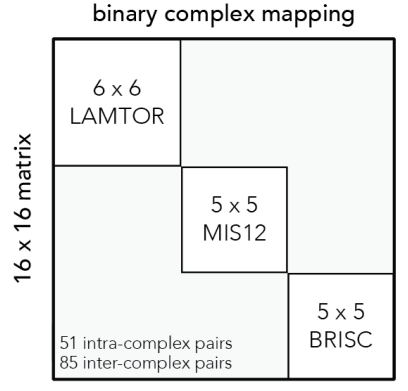

c

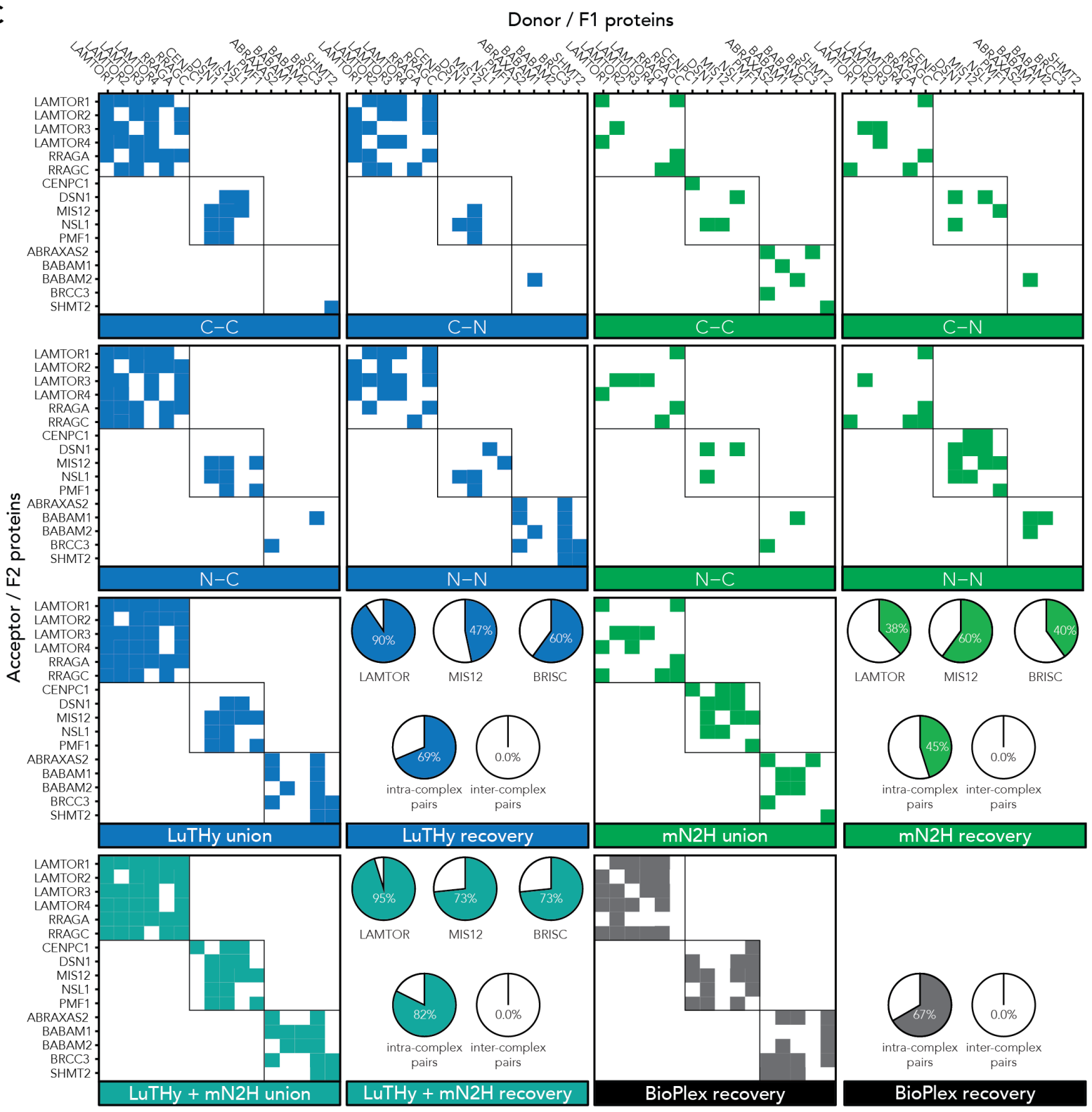


bioRxiv preprint doi: https://doi.org/10.1101/2021.08.25.457734; this version posted August 26,2021 . The copyright holder for this preprint (which was not certified by peer review) is the author/funder, who has granted bioRxiv a license to display the preprint in perpetuity. It is made available under aCC-BY-NC-ND 4.0 International license.

784 Figure 3. Mapping interactions within multiprotein complexes using the LuTHy and mN2H assays.

785 (A) Structures of the protein complexes analyzed in this study: LAMTOR (PDB: 6EHR), MIS12 (PDB: 5LSK), and 786 BRISC (PDB: 6H3C). (B) Binary interaction approach to systematically map PPIs within distinct complexes. Every protein subunit from each complex was screened against every other one (all-by-all, 16x16 matrix). (C) Results of

788 the all-by-all interaction screen for the selected multiprotein complexes. Each protein pair was systematically tested

789 in every possible configuration (i.e. C-C, C-N, N-C, N-N), and as donor and acceptor in LuTHy, or as F1 and F2

790 NanoLuc fusions in mN2H. LuTHy union corresponds to the combination of both LuTHy-BRET and LuTHy-LuC

791 results. Protein pairs tested in the LuTHy assays were scored positive if at least one of the two readouts (i.e.

792 LuTHy-BRET and/or LuTHy-LuC) was positive. LuTHy + mN2H union corresponds to the combination of results

793 from LuTHy union and $\mathrm{mN} 2 \mathrm{H}$ union. In the pie charts, the top panels show recovery rates of intra-complex pairs

794 within the LAMTOR, MIS12 and BRISC complexes, while the bottom panels show recoveries of all intra-complex

795 and inter-complex pairs. Published BioPlex results, corresponding to AP-MS data, were used for the interaction

796 matrix and pie charts. Proteins used as baits in BioPlex are indicated on the $\mathrm{x}$-axis, whereas identified interaction

797 partners are indicated on the y-axis. 
A

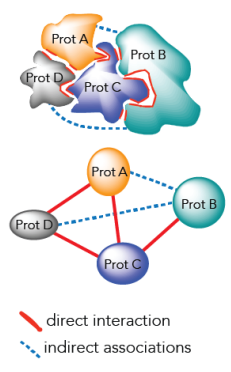

B

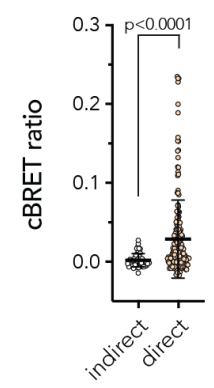

C

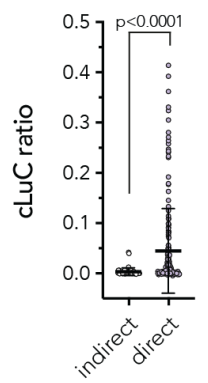

D

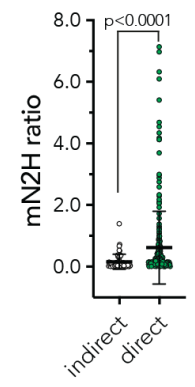

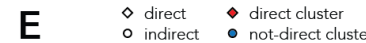

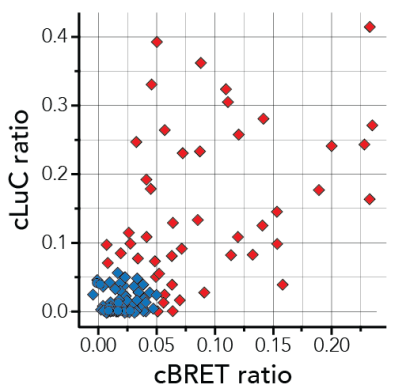

F

recovered direct interactions classified as indirect associations direct interactions

BioPlex (AP-MS) $0.0 \% 0.0 \%$

LuTHy + mN2H union

$0.0 \% \quad 67.7 \%$

LuTHy union

$0.0 \% 45.2 \%$

$\mathrm{mN} 2 \mathrm{H}$

$0.0 \% 48.4 \%$

G

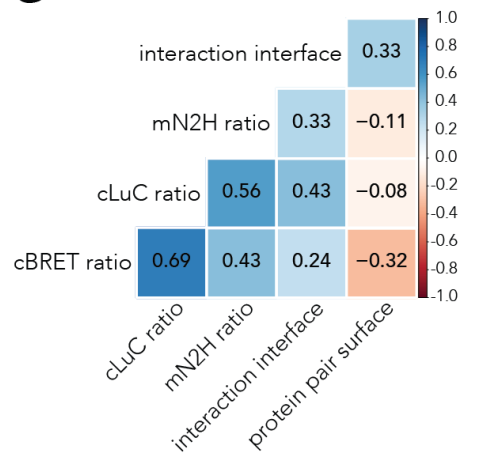

$\mathrm{H}$

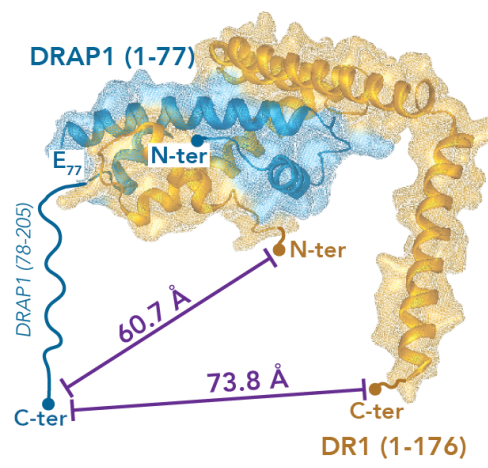

I

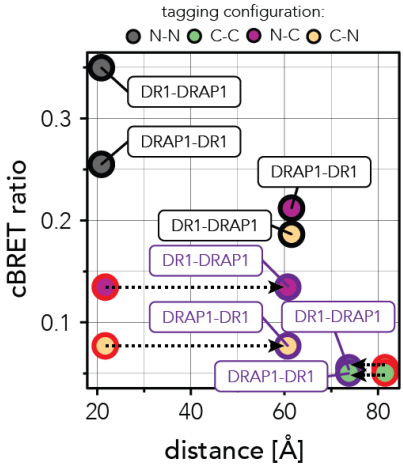

J

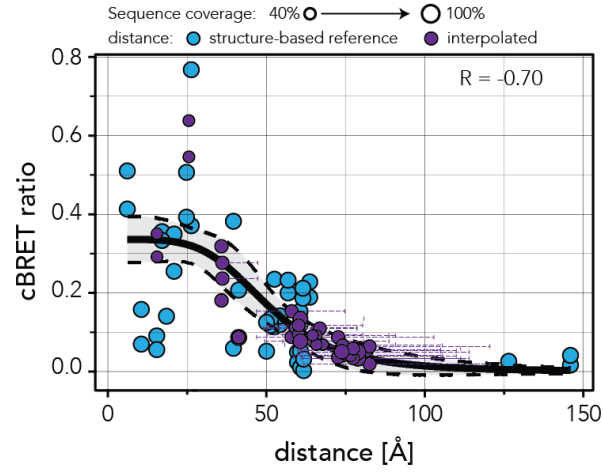

Figure 4. Differentiating direct interactions from indirect associations with LuTHy and mN2H assays.

800 (A) Schematic for the definition of direct interactions and indirect associations within complexes using structural biology data. (B-D) Distribution of the quantitative scores and comparison between direct interactions and indirect associations. Direct interactions produce significantly higher LuTHy-BRET (B), LuTHy-LuC (C), and $\mathrm{mN} 2 \mathrm{H}(\mathrm{D})$ scores compared to indirect associations (Welch's two-tailed t-test). (E) Scatter plot of cBRET and cLuC ratios showing direct interactions (diamonds) and indirect associations (circles) detected by LuTHy-BRET or LuTHy-LuC. Protein pairs were clustered by supervised expectation-maximization clustering as direct (red) or not-direct (blue) interactions using hsPRS-PDB and hsRRS-v2 as training sets. (F) Recovery and classification of structurally defined direct interactions as true direct interactions or as indirect associations by BioPlex (AP-MS), LuTHy and $\mathrm{mN} 2 \mathrm{H}$. (G) Pearson correlation matrix for direct interactions comparing cBRET, cLuC and $\mathrm{mN} 2 \mathrm{H}$ ratios to the interaction interface areas $\left(\AA^{2}\right)$, or to the total complex surface areas $\left(\AA^{2}\right)$. $(\mathbf{H}) 3 D$ structure of the DR1-DRAP1 
810 interaction (PDB: 1JFI). Full-length DR1 but only the N-terminal region of DRAP1 (1-77, amino acids 78-205

811 lacking) are reported in the 3D structure. The CBRET ratios were used to interpolate distances between the C-

812 terminus of DRAP1 and the N-terminus (60.7 $\AA$ ), or C-terminus (73.8 $\AA$ ) of DR1 based on the cBRET-distance

813 standard curve. (I) CBRET ratios for the DR1-DRAP1 PPI are plotted against the molecular distances obtained

814 from the 3D structure. Tagging configurations are colored by N-N (grey), C-C (green), N-C (purple) or C-N (yellow).

815 Each data point on the graph is labeled (framed text) according to the tested tagging configuration: the protein

816 indicated first is tagged with NanoLuc (NL) luciferase, while the second protein is tagged with PA-mCitrine (PA-

$817 \mathrm{mCit}$ ) (e.g. DR1+DRAP1 (N-N, grey) corresponds to NL-DR1/PA-mCit-DRAP1). Tagging configurations where tags

818 are fused to the structurally unresolved termini in the current 3D structure for one of the two proteins are outlined

819 in red (e.g. DR1+DRAP1 (N-C, purple) corresponds to NL-DR1/DRAP1-mCit-PA). Interaction values outlined in

820 purple represent cBRET ratios against interpolated molecular distances. Differences between structurally

821 determined and interpolated distances are indicated by dotted arrows. (J) cBRET ratios plotted against the

822 structurally determined molecular distances for the 44 protein pairs used as references (blue). The 30 PPls with

823 tags fused to protein termini not currently resolved in the structures are plotted against interpolated distances

824 between full-length proteins (purple). For each tested pair, the purple horizontal error bar corresponds to the 95\%

825 confidence interval of the interpolated molecular distances. Sigmoidal fits were performed on the 44 tested pairs outlined in black, together with the 30 pairs outlined in purple $(R=-0.70)$. 
A

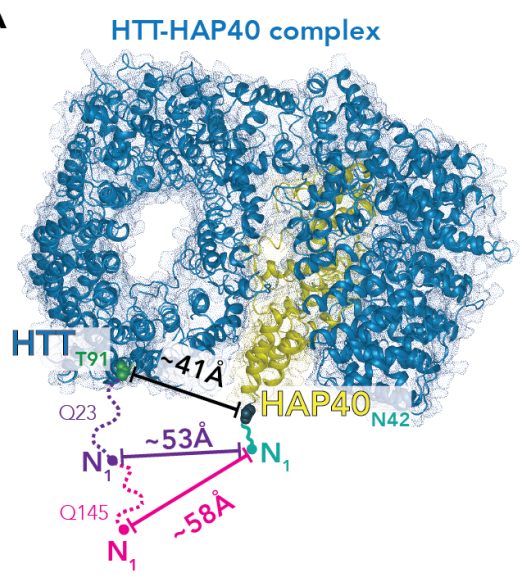

B

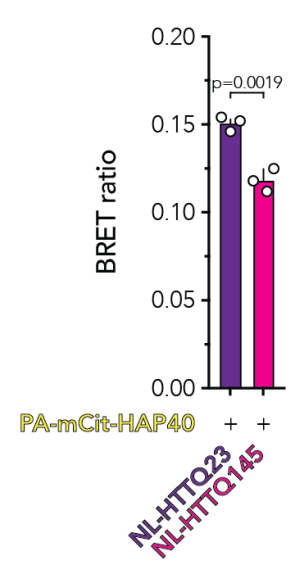

$E$

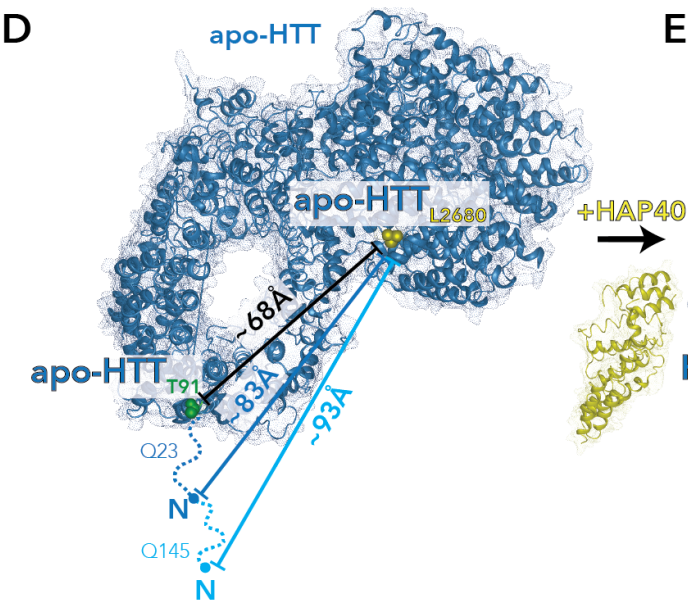

HTT-HAP40 complex

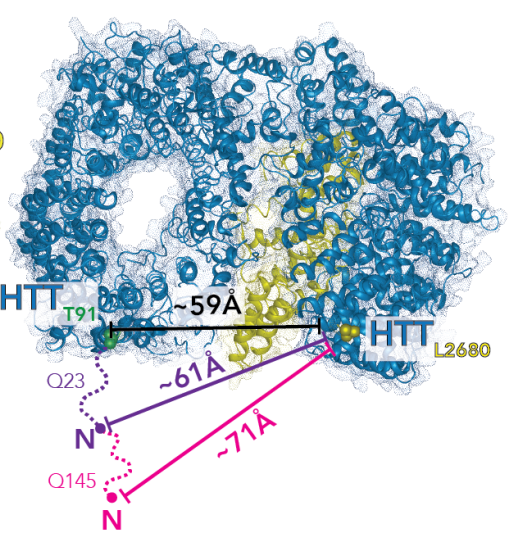

C

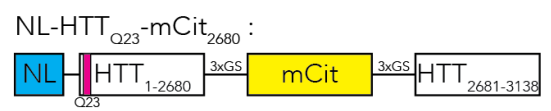

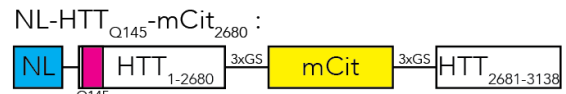

\section{Figure 5. Interpolating molecular distances within the HTT-HAP40 complex with LuTHy-BRET.}

(A) Cryo-EM structure of the HTT-HAP40 complex (PDB: 6EZ8). The distances between HTT(T91) and HAP40(N42) inferred from the structure are indicated (black), along with the distances between the N-terminal domains of HAP40 and HTTQ23 (purple) or HTTQ145 (magenta) interpolated from the measured BRET ratios. (B)

832 The BRET ratio between HAP40 and HTTQ23 is significantly higher than the one between HAP40 and HTTQ145

833 (the bar plots show means \pm standard deviations (sd), statistical significance was calculated by a two-tailed t-test, n=3). (C) Schematic representations of the HTTQ23 and HTTQ145 intramolecular LuTHy-BRET sensors. (D,E)

835 Cryo-EM structures of the apo-HTT protein (D, PBD: 6RMH) and the HTT-HAP40 complex (E, PDB: 6EZ8) where 836 the distances between residues T91 and L2680 are indicated (black) either in the absence or presence of HAP40.

837 (F) BRET ratios measured for the intramolecular LuTHy-BRET sensors in the absence or presence of HAP40, for

$838 \mathrm{HTTQ} 23$ and HTTQ145 (the bar plots show means \pm sd, statistical significance was calculated by two-way ANOVA

839 with Tukey's multiple comparisons test, $n=3$ ). The BRET ratios were used to interpolate distances between the $\mathrm{N}$ terminus of HTT and residue L2680 for HTTQ23 and HTTQ145, which are indicated in D and E. 
A

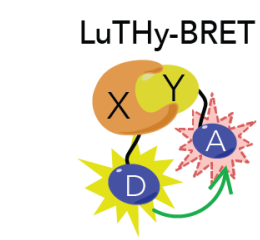

LuTHy-LuC

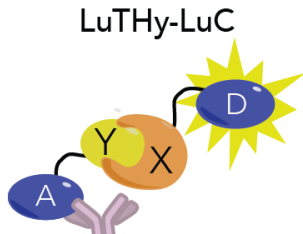

(AB)
B

Tagging configurations

$\begin{array}{lll}\text { N1-X / N2-Y: } & \text { NL_ProteinX / PA-mCit_ProteinY } \\ \text { N1-X / C2-Y: } & \text { NL_ProteinX / ProteinY_PA-mCit } \\ \text { C1-X / N2-Y: } & \text { ProteinX-NL / PA-mCit_ProteinY } \\ \text { C1-X / C2-Y: } & \text { ProteinX-NL / ProteinY_PA-mCit } \\ \text { N1-Y / N2-X: } & \text { NL_ProteinY / PA-mCit_ProteinX } \\ \text { N1-Y / C2-X: } & \text { NL_ProteinY / ProteinX-PA-mCit } \\ \text { C1-Y / N2-X: } & \text { ProteinY-NL / PA-mCit_ProteinX } \\ \text { C1-Y / C2-X: } & \text { ProteinY-NL / ProteinX-PA-mCit }\end{array}$

C1-Y / C2-X:

Protein Y-NL

Protein $\mathbf{X}-$ PA-mCit

C

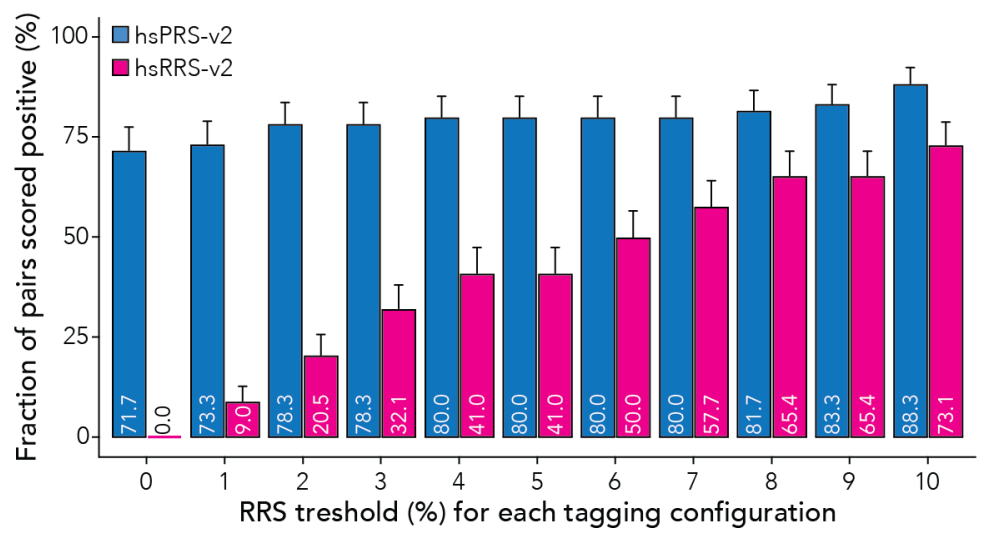

842 Supplementary Figure 1 (related to Figure 1). Tagging configurations and relationship between the hsRRS-

843 v2 threshold and detection of hsPRS-v2 and hsRRS-v2 pairs for LuTHy.

844 (A) Schematic overview of the LuTHy-BRET and LuTHy-LuC assays. X: Protein X, Y: Protein Y, D: NanoLuc donor,

845 A: mCitrine acceptor, AB: antibody. (B) With the LuTHy assay, each protein pair X-Y can be tested in four possible

846 configurations ( $\mathrm{N}$ - vs. C-terminal fusion for each protein), and proteins can be swapped from one tag to the other

847 for a total of 8 possible LuTHy versions resulting in 16 quantitative scores for each protein pair (i.e. eight for LuTHy-

848 BRET and eight for LuTHy-LuC). (C) Impact of scoring random protein pairs on PPI recovery for all LuTHy versions:

849 cumulative detection rates of hsPRS-v2 and hsRRS-v2 pairs when increasing, identical hsRRS-v2 thresholds are

850 applied for each individual assay version. Error bars in (C) indicate standard errors of the proportion. 
bioRxiv preprint doi: https://doi.org/10.1101/2021.08.25.457734; this version posted August 26, 2021. The copyright holder for this preprint

(which was not certified by peer review) is the author/funder, who has granted bioRxiv a license to display the preprint in perpetuity. It is made available under aCC-BY-NC-ND 4.0 International license.

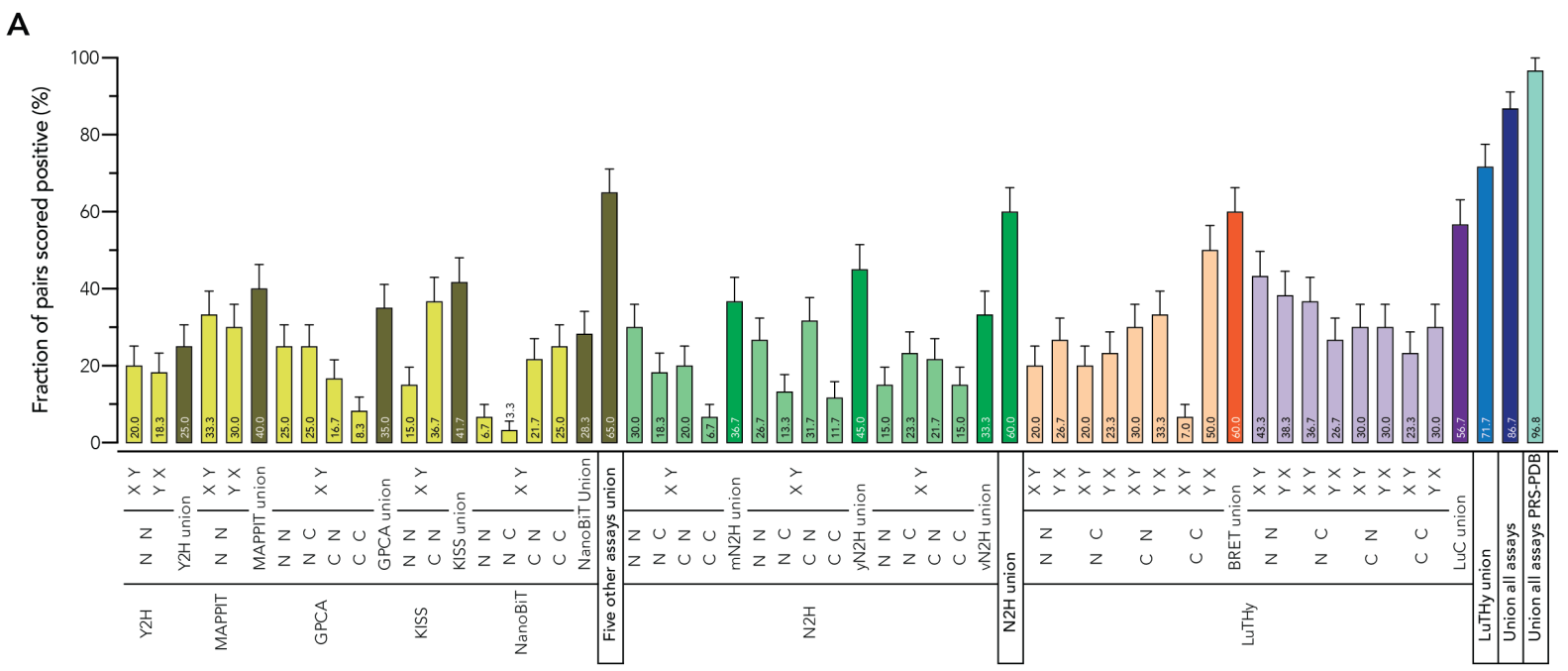

B

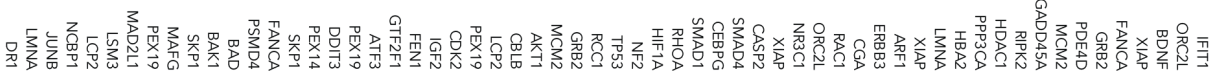

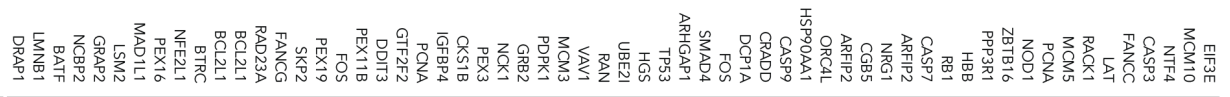

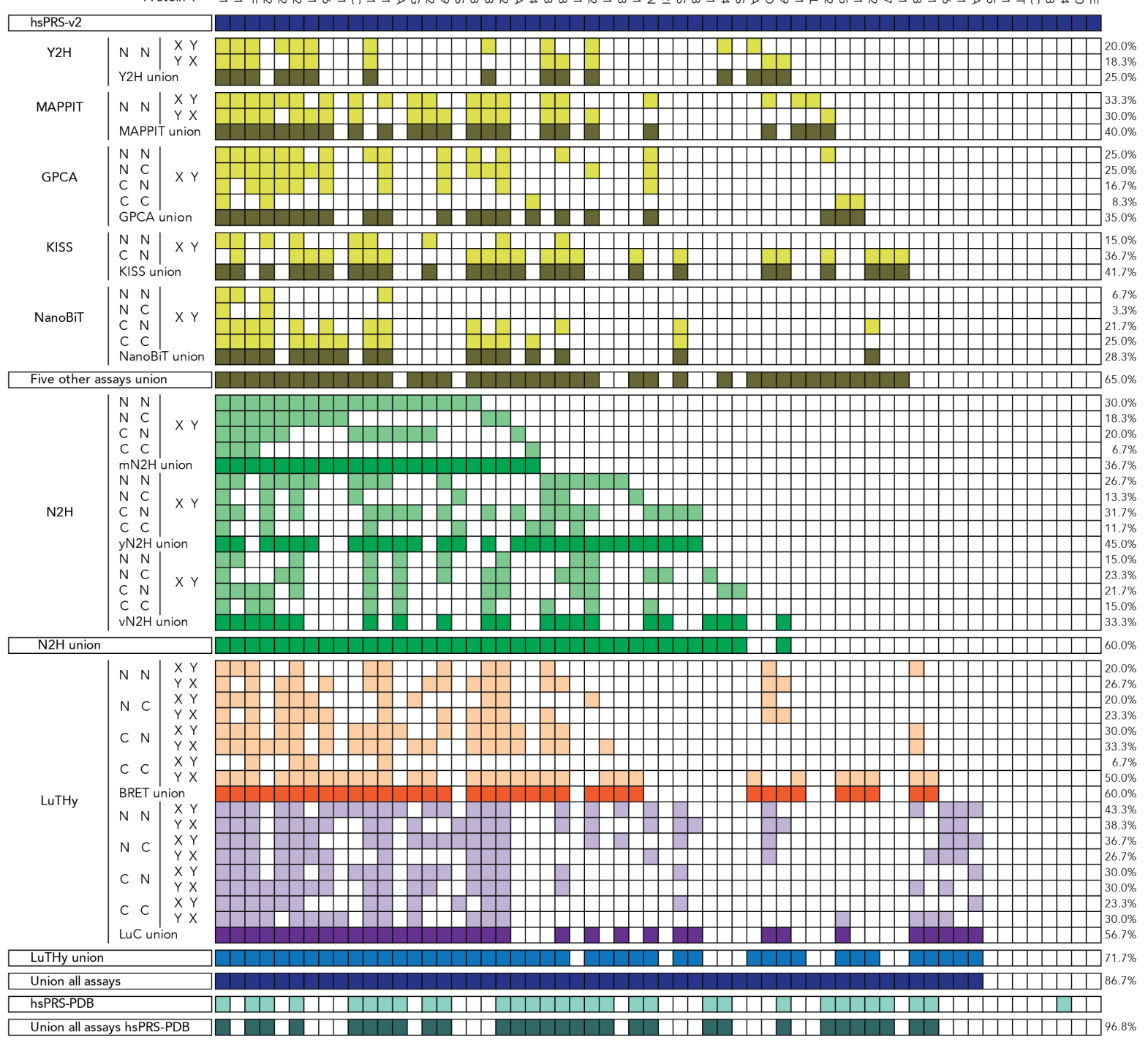


bioRxiv preprint doi: https://doi.org/10.1101/2021.08.25.457734; this version posted August 26, 2021. The copyright holder for this preprint

(which was not certified by peer review) is the author/funder, who has granted bioRxiv a license to display the preprint in perpetuity. It is made available under aCC-BY-NC-ND 4.0 International license.

852 Supplementary Figure 2 (related to Figure 1). Performance and complementarity of different binary PPI

853 assay versions under conditions where none of the hsRRS-v2 pairs are scored positive.

854 (A,B) Performance of individual binary PPI assay versions benchmarked in Choi et al ${ }^{18}$ (Y2H, MAPPIT, GPCA,

855 KISS, NanoBiT, N2H), and in this study (LuTHy). In total, 42 assay versions were tested, reaching $~ 87 \%$ detection

856 of hsPRS-v2 PPIs. The 31 hsPRS-PDB pairs correspond to hsPRS-v2 PPIs currently supported by at least one 3D

857 structure in PDB. The percentage (\%) at the end of each row represents the fraction of hsPRS-v2 PPIs scored

858 positive when none of the hsRRS-v2 pairs are recovered. Error bars in $(\mathbf{A})$ indicate standard errors of the

859 proportion. 
A

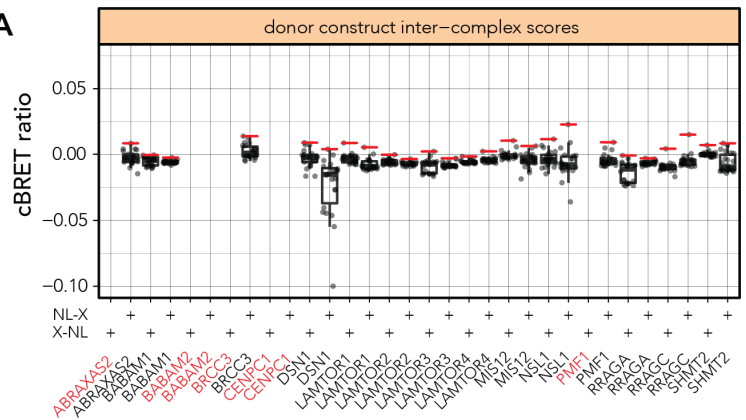

Donor proteins

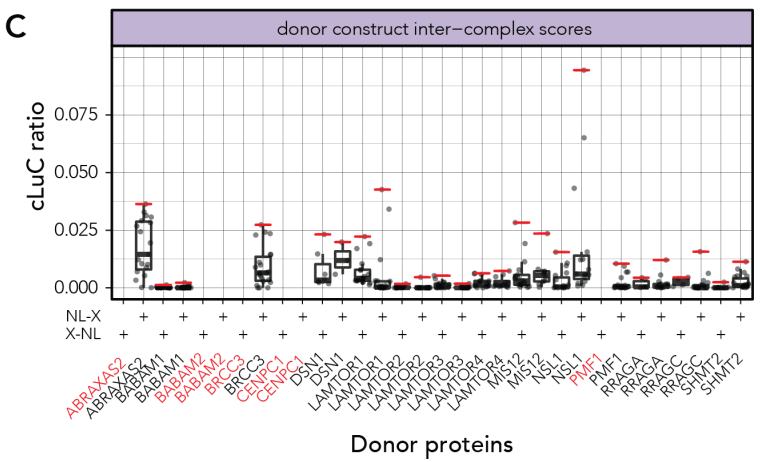

$\mathrm{E}$

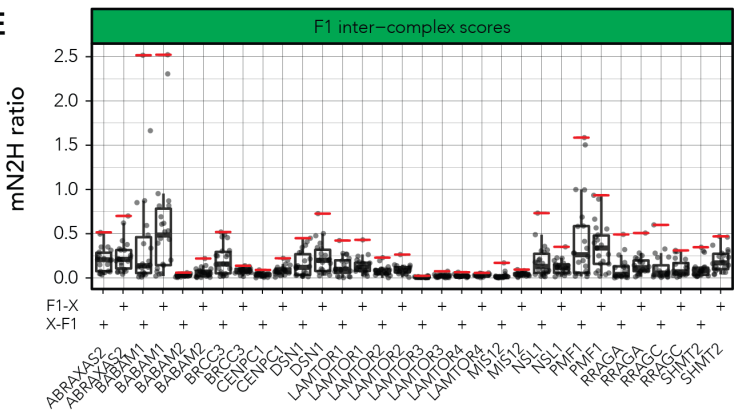

F1 proteins

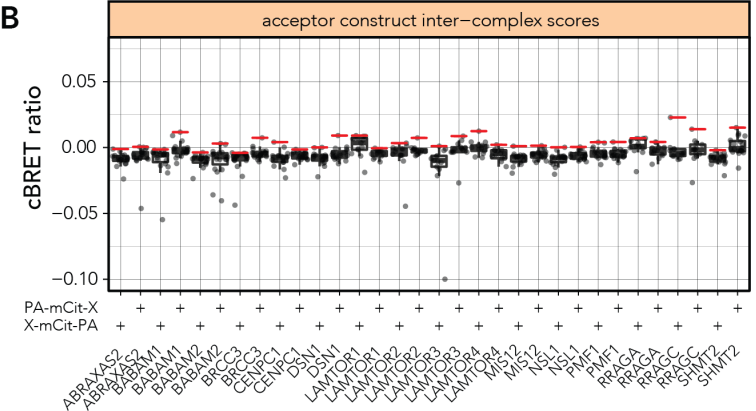

Acceptor proteins

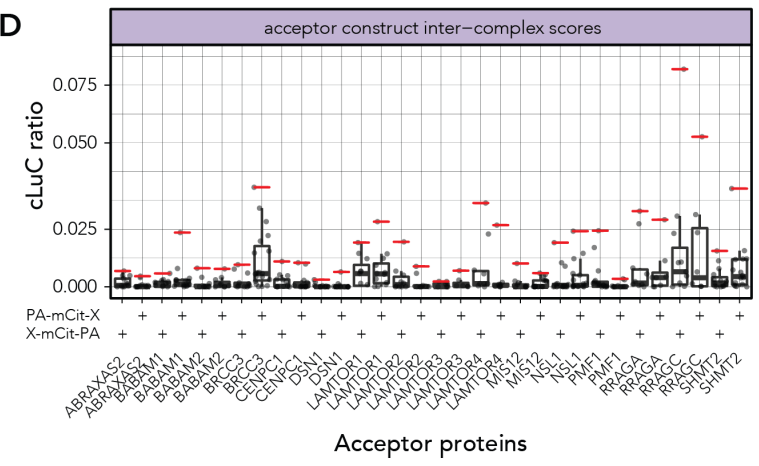

F

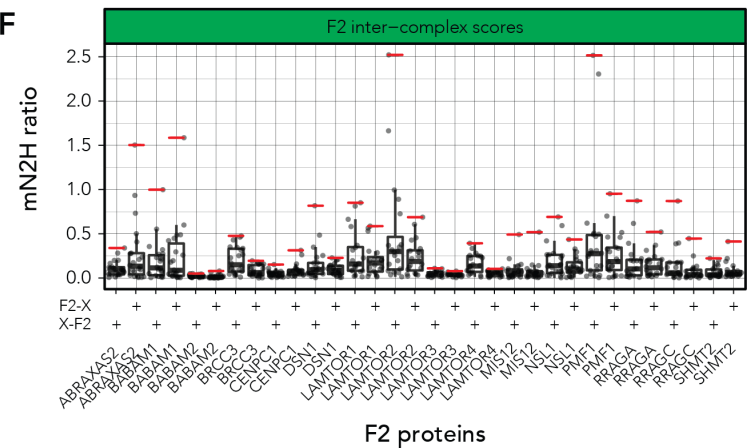

Supplementary Figure 3 (related to Figure 3). Establishing a construct-specific scoring threshold.

For each assay and expression construct, boxes and whiskers for the quantitative scores of inter-complex pairs are shown (displayed are the median, lower and upper hinges showing $25^{\text {th }}$ and $75^{\text {th }}$ percentiles, lower and upper whiskers extending from the hinges with $1.5 x$ the inter-quartile range). Individual scores are indicated by small grey dots. The horizontal red lines indicate the cutoffs applied for each construct. Constructs written in red on the x-axes showed no detectable expression. Scores of inter-complex pairs differentiated by individual constructs for LuTHy$\operatorname{BRET}(\mathbf{A}, \mathbf{B})$, LuTHy-LuC $(\mathbf{C}, \mathbf{D})$, and $\mathrm{mN2H}(\mathbf{E}, \mathbf{F})$ assays. Each protein pair was tested in every possible configuration (i.e. C-C, C-N, N-C, N-N), and each protein was also tested as donor (A, C) and acceptor (B, D) in LuTHy, or as F1 (E) and F2 (F) NanoLuc fusions in mN2H. The (+) indicates the $\mathrm{N}$ - or C-terminal tagging configuration of the indicated protein. 


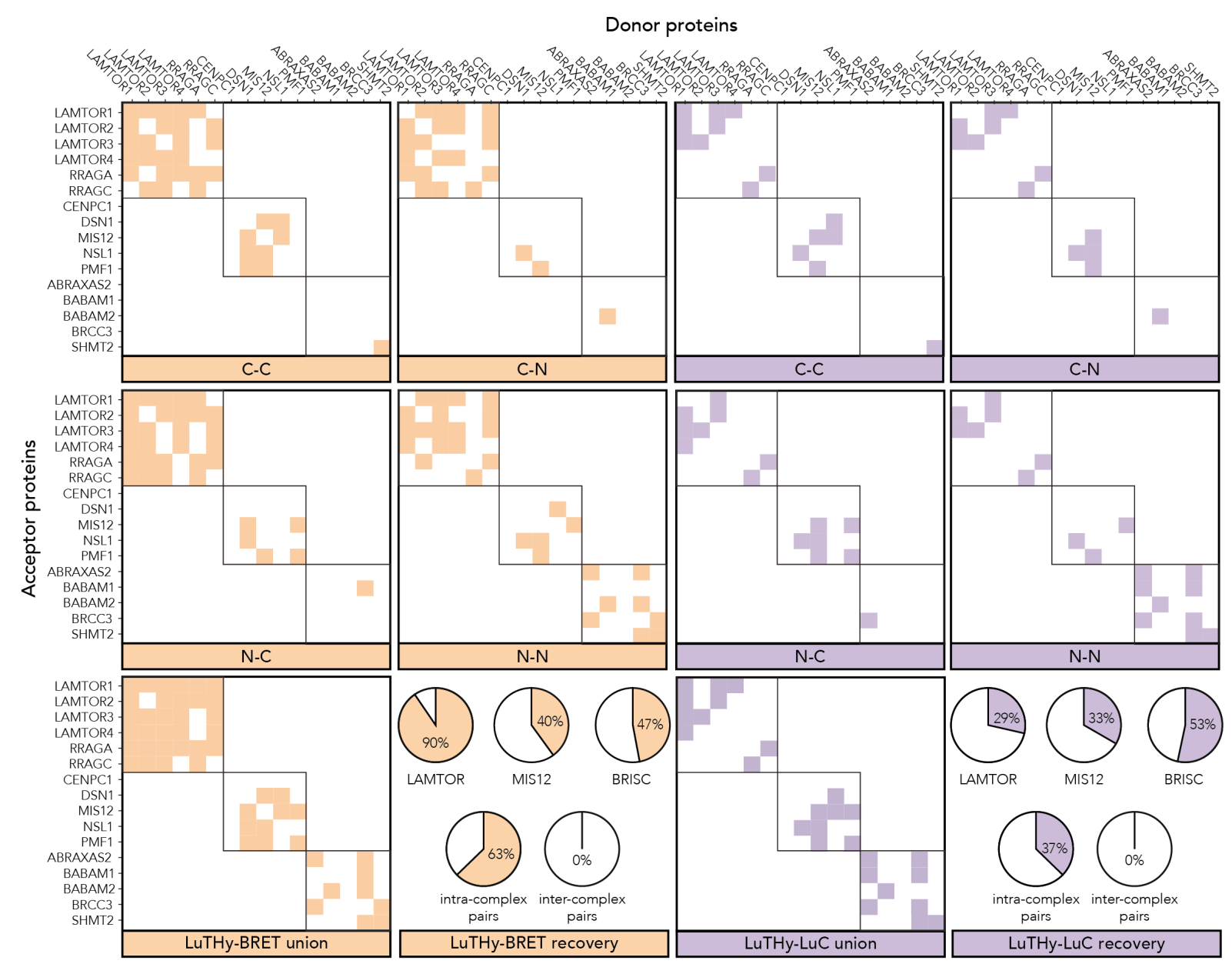

872 Supplementary Figure 4 (related to Figure 3). Mapping interactions within multiprotein complexes using

\section{3 the LuTHy-BRET and LuTHy-LuC assays.}

874 Results of the all-by-all interaction screen with the LuTHy-BRET and LuTHy-LuC assays for the selected

875 multiprotein complexes. Each protein pair was tested in every possible configuration (i.e. C-C, C-N, N-C, N-N), and

876 each protein was also tested as donor and acceptor. In the pie charts, the top panels show the recovery rates of 877 intra-complex pairs within the LAMTOR, MIS12 and BRISC complexes, while the bottom panels show the recovery rates of all intra-complex and inter-complex pairs. 
A

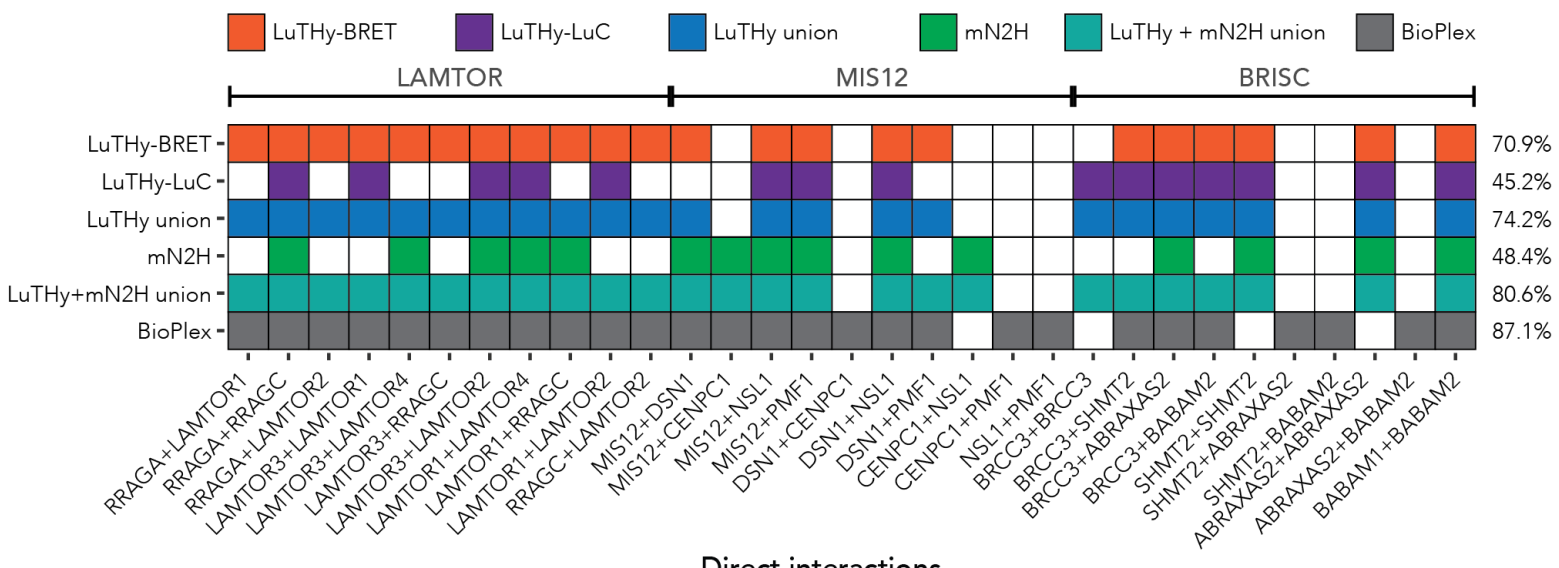

B

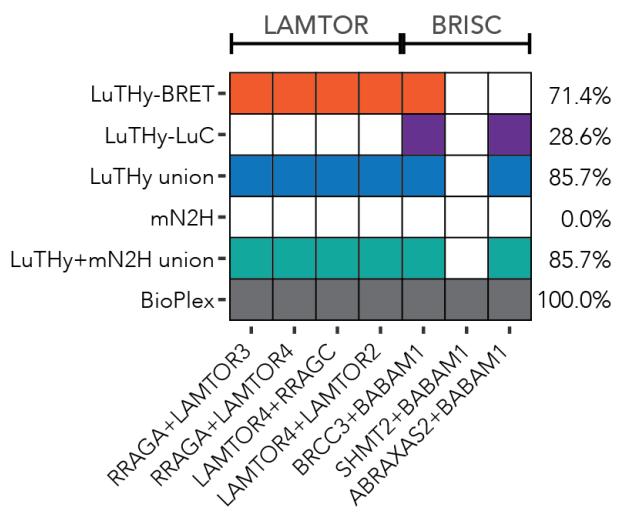

Indirect associations
C

\begin{tabular}{lr|l} 
& $\begin{array}{r}\text { recovered indirect associations classified as } \\
\text { indirect associations }\end{array}$ & direct interactions \\
BioPlex (AP-MS) & $0.0 \%$ & $0.0 \%$ \\
LuTHy $+\mathrm{mN} 2 \mathrm{H}$ union & $0.0 \%$ & $0.0 \%$ \\
LuTHy union & $0.0 \%$ & $0.0 \%$ \\
$\mathrm{mN} 2 \mathrm{H}$ & $0.0 \%$ & $0.0 \%$
\end{tabular}
associations detected by different approaches.

882 Detected direct interactions $(\mathbf{A})$ and indirect associations $(\mathbf{B})$ by the LuTHy and mN2H binary PPI assays, and by 883 AP-MS-based (BioPlex) techniques. The percentage (\%) at the end of each row represents the fraction of protein 884 pairs recovered (for LuTHy and $\mathrm{mN} 2 \mathrm{H}$ : at a threshold where no inter-complex pair is scored positive). LuTHy + $885 \mathrm{mN} 2 \mathrm{H}$ union summarizes the LuTHy and $\mathrm{mN} 2 \mathrm{H}$ results. Published reference data from the AP-MS BioPlex dataset are shown. Structural biology data were used to define direct interactions and indirect associations. (C) Recovery and classification of structurally defined indirect associations as true indirect associations or as direct interactions for BioPlex, LuTHy and mN2H. 
A

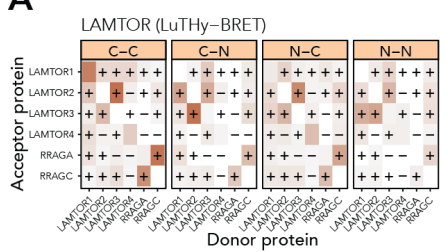

D

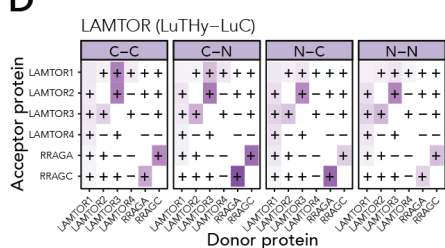

G

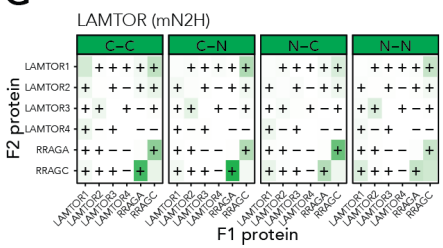

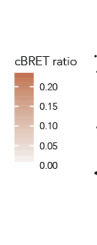

$\mathrm{E}$

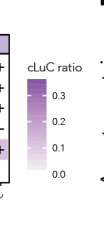

$\mathrm{H}$

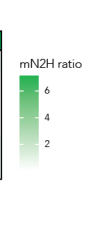

B

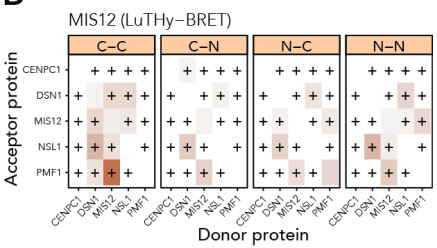

E

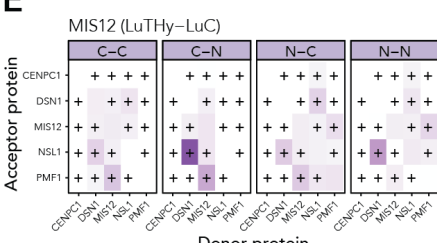

H

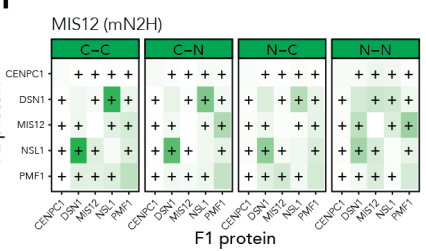

C

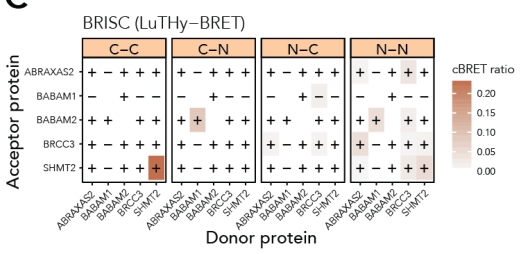

$\mathbf{F}$

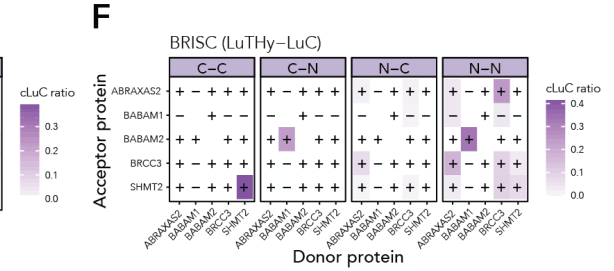

I

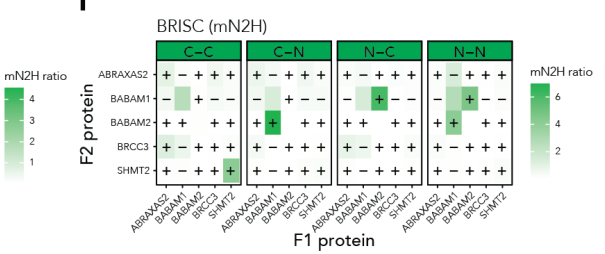

890 Supplementary Figure 6 (related to Figure 4). Quantitative scores for direct interactions and indirect 891 associations according to the tested tagging configurations.

892 Heat maps of all intra-complex protein pairs in the LAMTOR, MIS12 and BRISC complexes, differentiated by

893 tagging configurations for LuTHy-BRET (A-C), LuTHy-LuC (D-F) and mN2H (G-I). The range of quantitative scores

894 is depicted from smaller, or equal to zero (white) to maximal (orange, purple or green for LuTHy-BRET, LuTHy-

895 LuC or mN2H, respectively) values. A "+" symbol indicates a direct interaction, a "-" symbol an indirect association, and an empty cell a separate homodimer interaction. 
A

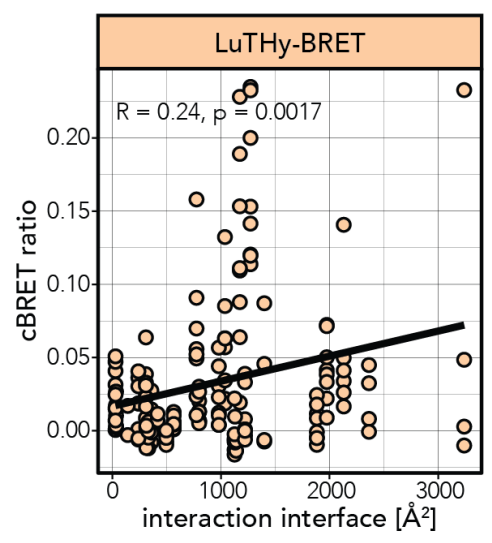

D

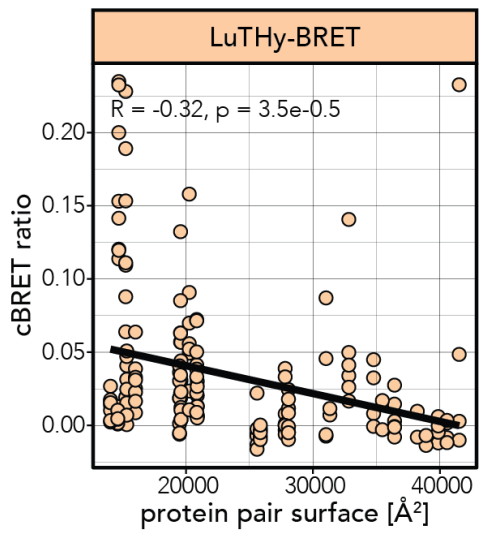

G

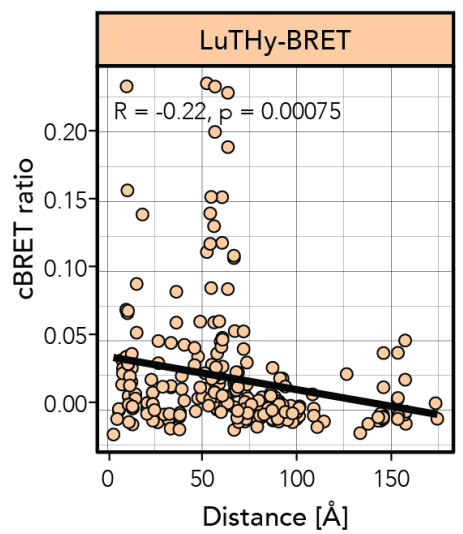

B

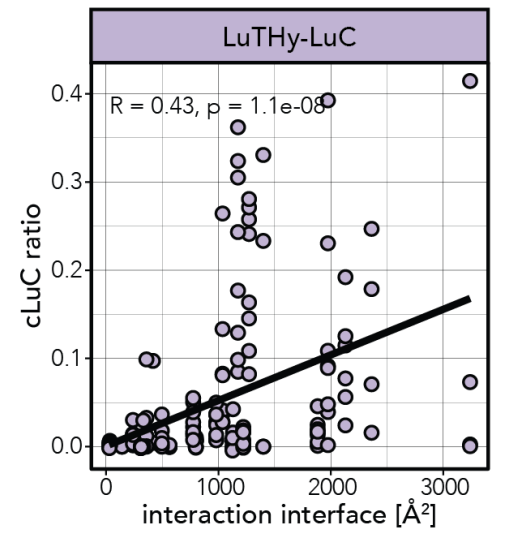

E

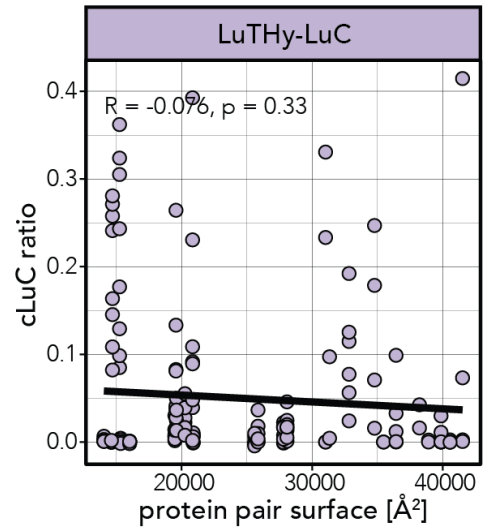

$\mathrm{H}$

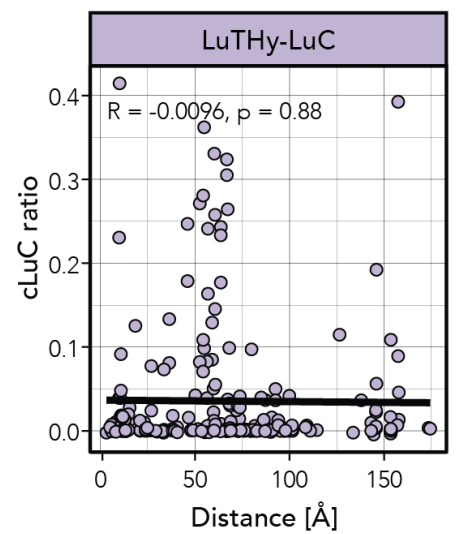

C

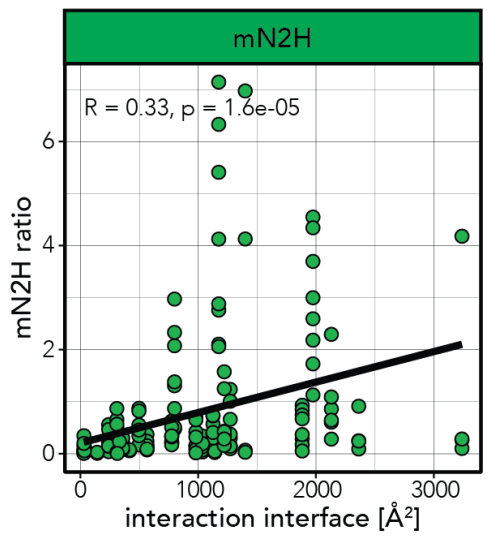

F

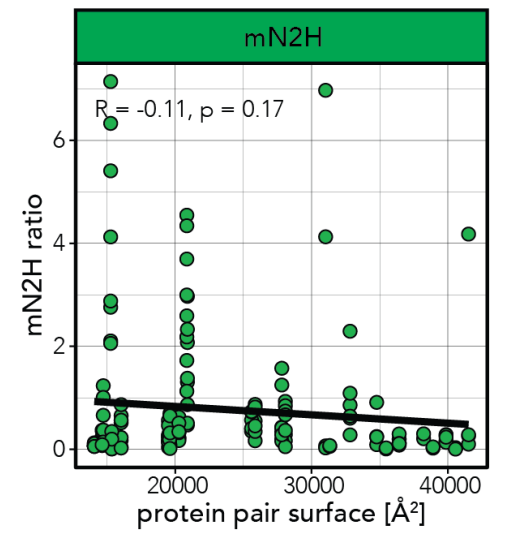

I

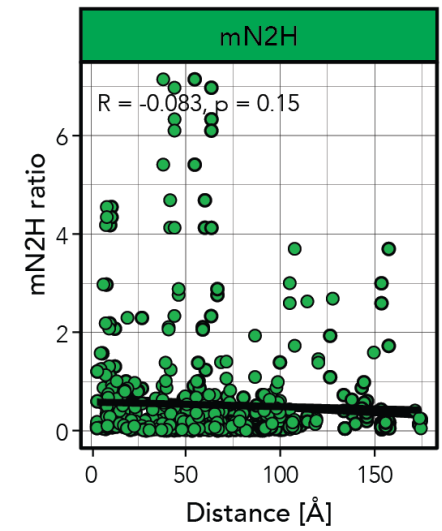

J

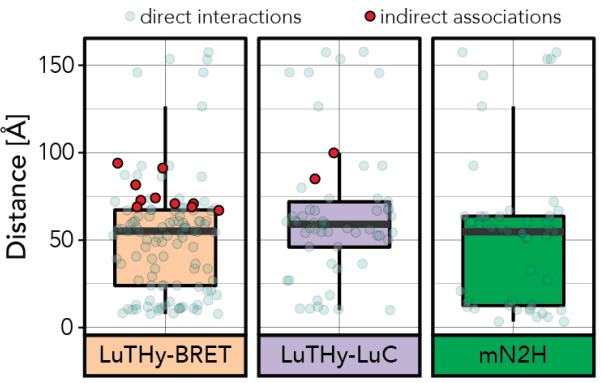


898 Supplementary Figure 7 (related to Figure 4). Correlation between the LuTHy and mN2H quantitative

899 scores and the structural properties of the analyzed complexes.

900 Correlation between the interaction interfaces between proteins and the cBRET (A), cLuC (B), and mN2H (C) ratios

901 for the corresponding PPIs. Correlation between the total surface areas of interacting proteins and the cBRET (D),

$902 \mathrm{cLuC}(\mathbf{E})$, and $\mathrm{mN} 2 \mathrm{H}(\mathbf{F})$ ratios. Correlation between the molecular distances $(\AA)$ of the tagged protein termini and

903 the cBRET $(\mathbf{G})$, cLuC $(\mathbf{H})$, and $\mathrm{mN} 2 \mathrm{H}(\mathbf{I})$ ratios of the corresponding PPIs. Pearson correlation coefficients (R) with

904 p-values are displayed. (J) Box plots of the molecular distances ( $\AA$ ) between the tagged protein termini for the

905 detected direct interactions (transparent green) and indirect associations (red) in the LuTHy-BRET, LuTHy-LuC

906 and $\mathrm{mN} 2 \mathrm{H}$ assays (displayed are the median, lower and upper hinges showing the $25^{\text {th }}$ and $75^{\text {th }}$ percentiles, lower

907 and upper whiskers extending from the hinges with $1.5 x$ the inter-quartile range). 
A
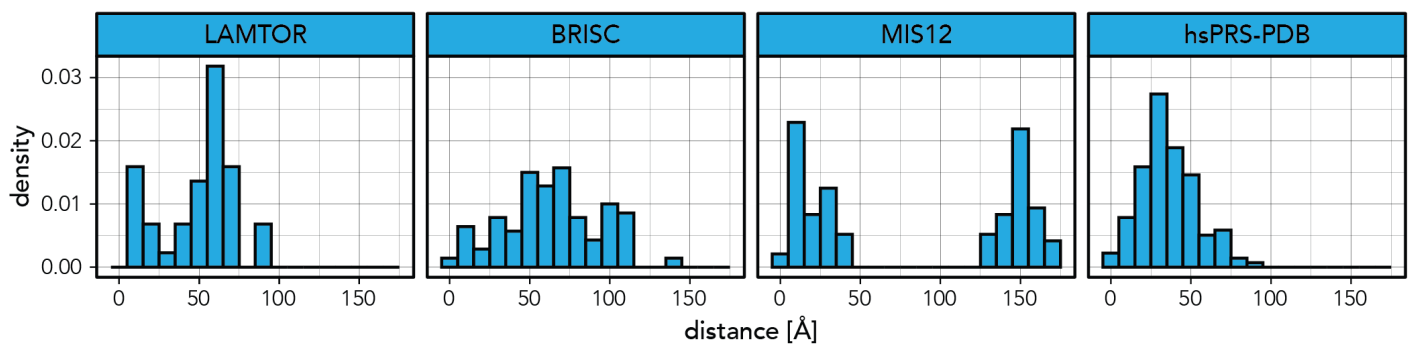

B
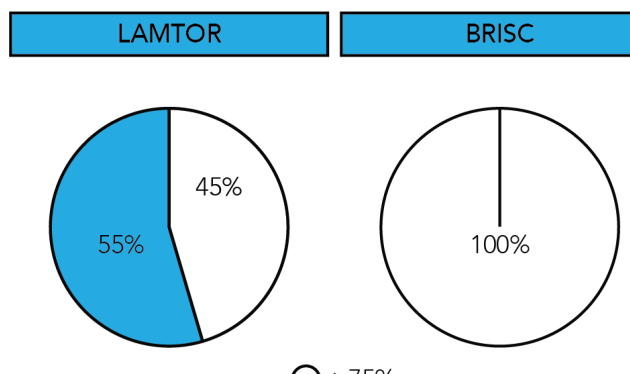

$\bigcirc>75 \%$ sequence coverage
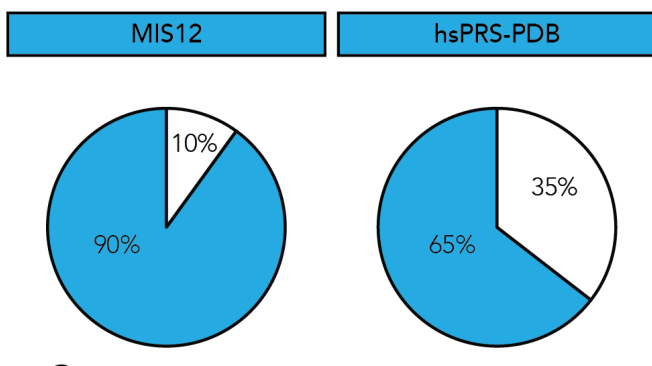

$<75 \%$ sequence coverage

C

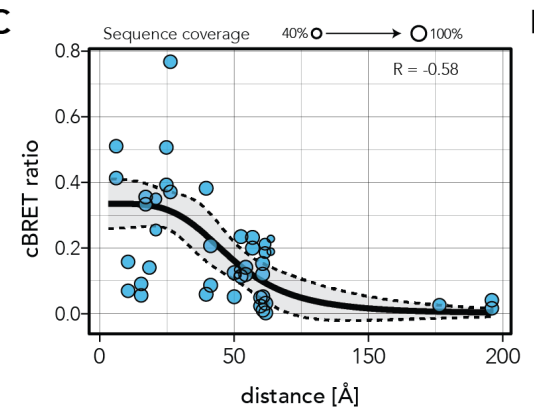

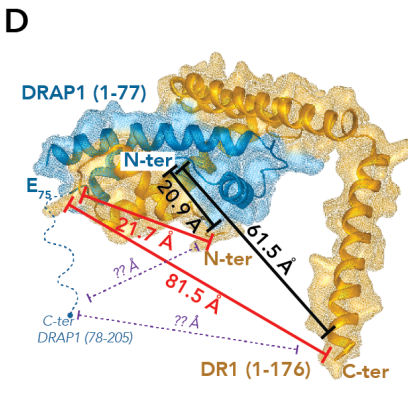

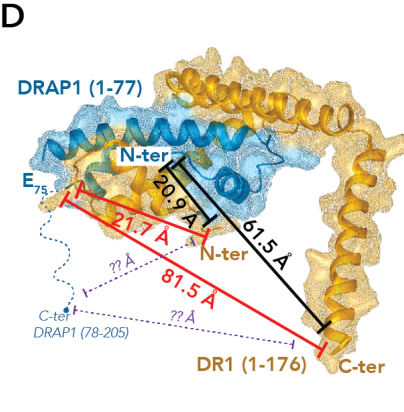

$\mathbf{F}$

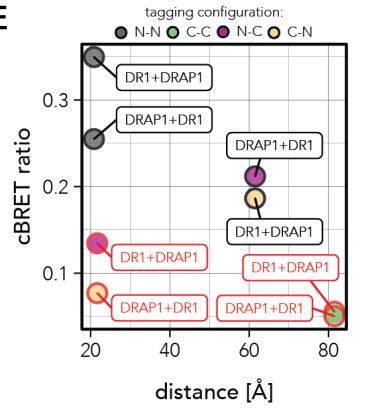

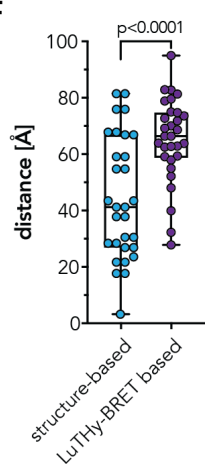
subunits of the studied complexes used to generate the cBRET-distance standard curve.

911 (A) Relative distribution of structure-based distances for direct interactions within the LAMTOR, BRISC and MIS12 complexes, as well as in the hsPRS-PDB PPIs. (B) Proportion of directly interacting proteins reported in the 3D structures that contain, on average, more (white) or less (green) than $75 \%$ of the full-length protein sequences. (C)

914 Correlation between the cBRET ratios and the molecular distances for the 44 protein pairs where at least six

915 tagging configurations out of the eight tested are scored positive in LuTHy-BRET, and for which the respective

916 tagged protein termini are structurally resolved. Goodness of the sigmoidal regression fit is indicated on the graph

$917(R=-0.58)$. (D) 3D structure of the DR1-DRAP1 interaction where molecular distances between the last amino

918 acids of the structurally solved proteins are indicated in red. The dotted blue lines indicate the protein fractions that

919 are not structurally resolved (DRAP1 C-terminus). Unknown molecular distances between the missing termini in the 3D structure are indicated by dotted purple lines. (E) cBRET ratios for the DR1-DRAP1 interaction are plotted against the structure-based molecular distances. Tagging configurations are colored by N-N (grey), C-C (green),

$922 \mathrm{~N}-\mathrm{C}$ (purple) or C-N (yellow). Each data point on the graph is labeled (framed text) according to the tested tagging 
923 configuration: the protein indicated first is tagged with NanoLuc (NL) luciferase, while the second protein is tagged

924 with PA-mCitrine (PA-mCit) (e.g. DR1+DRAP1 (N-N, grey) corresponds to NL-DR1/PA-mCit-DRAP1). Tagging

925 configurations where tags are fused to the structurally unresolved termini in the current 3D structure for one of the

926 two proteins are outlined in red (e.g. DR1+DRAP1 (N-C, purple) corresponds to NL-DR1/DRAP1-mCit-PA). (F)

927 Structure-based and predicted distances for the 30 PPIs with tags fused to protein termini not currently resolved in

928 the structures (box and whisker visualizing the median, lower and upper hinges showing the $25^{\text {th }}$ and $75^{\text {th }}$

929 percentiles, lower and upper whiskers extending from min to max; all data points are shown). Statistical significance

930 was calculated by a two-tailed, paired t-test, $n=30$. 
A

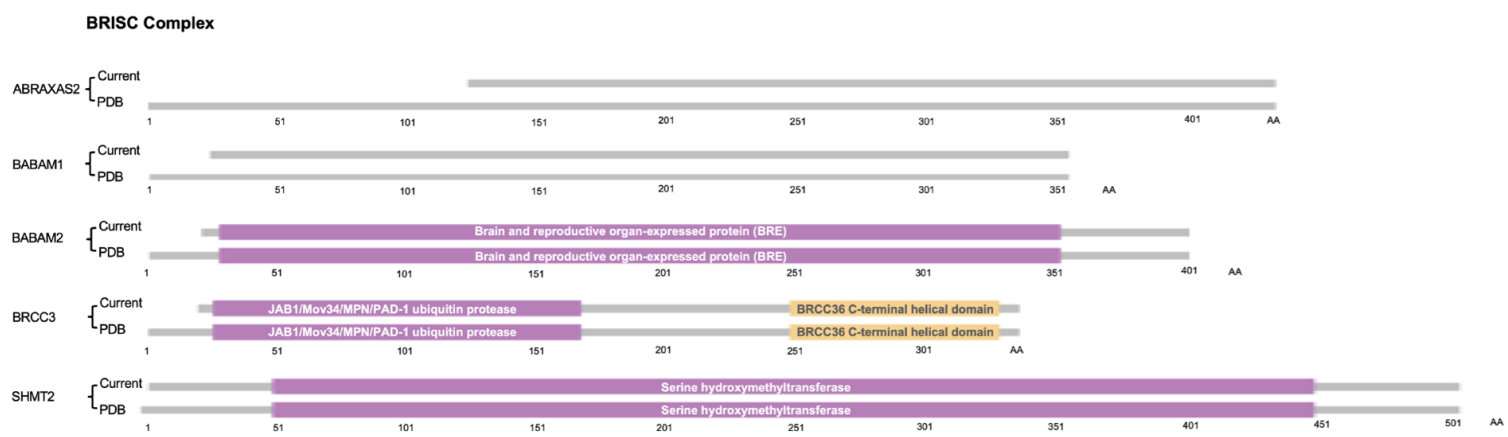

B

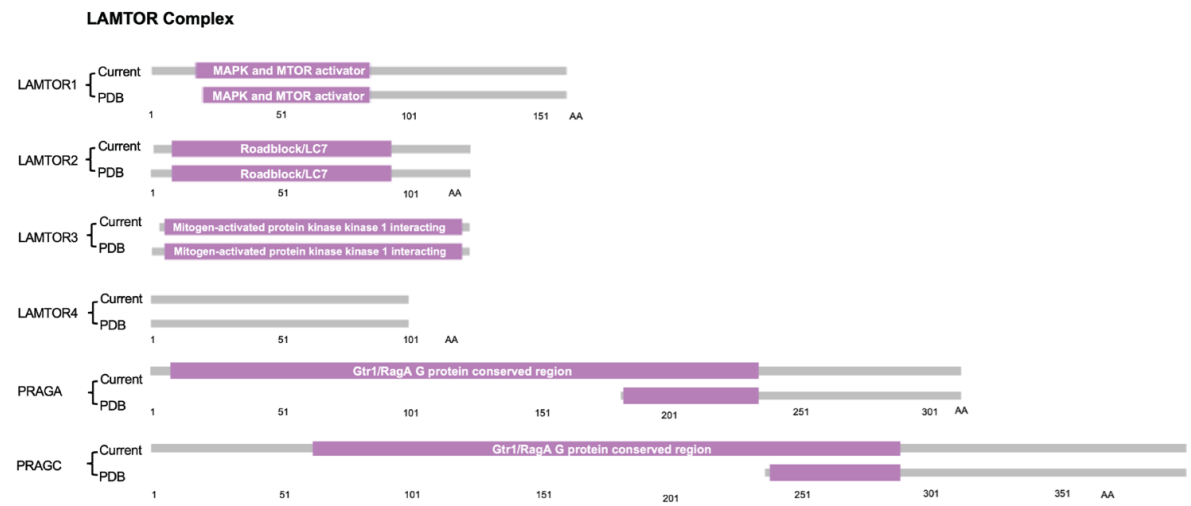

C

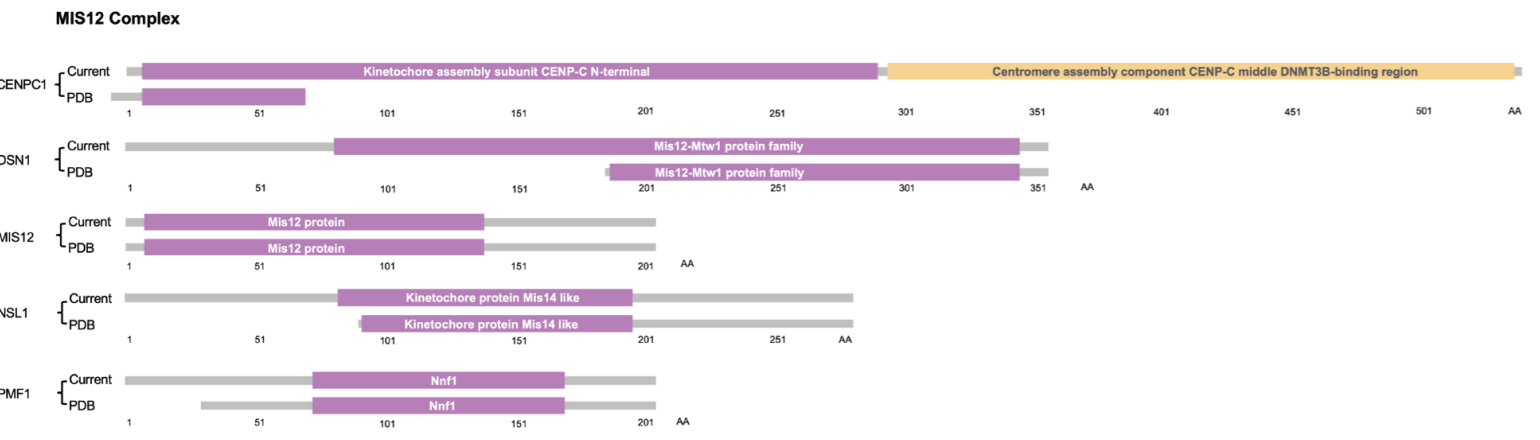
present binary interaction study (“Current”) compared to those found in the published structures (“PDB”).

934 Schematic representations of the protein constructs used in the BRISC (A), LAMTOR (B), and MIS12 (C) 935 complexes. The numbers indicate the lengths of the protein constructs in amino acids (AA). (A) The structural study characterizing the BRISC complex (PDB: $6 \mathrm{H} 3 \mathrm{C}$ ) used full-length proteins whereas four of the constructs (ABRAXAS2, BABAM1, BABAM2, BRCC3) used in the current study are C-terminally truncated. The current binary interaction study uses proteins containing $>90 \%$ of the BABAM1, BABAM2 and BRCC 3 , and $\sim 80 \%$ of the ABRAXAS2 full-length reference sequences. (B) The structural study characterizing the LAMTOR complex (PDB: 6EHR) uses smaller fragments for the RRAGA and RRAGC subunits. Except for a single AA missing on the N- 
bioRxiv preprint doi: https://doi.org/10.1101/2021.08.25.457734; this version posted August 26, 2021. The copyright holder for this preprint

(which was not certified by peer review) is the author/funder, who has granted bioRxiv a license to display the preprint in perpetuity. It is made available under aCC-BY-NC-ND 4.0 International license.

941 terminus of LAMTOR3, the current study uses the full-length sequences for subunits of the LAMTOR complex. (C)

942 Overall, the structural study of the MIS12 complex (PDB: 5 LSJ) lacks $>50 \%$ of the AA sequences for the different

943 subunits. 


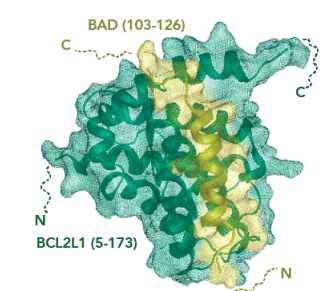

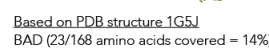

BC21211168/233 3 mino a d
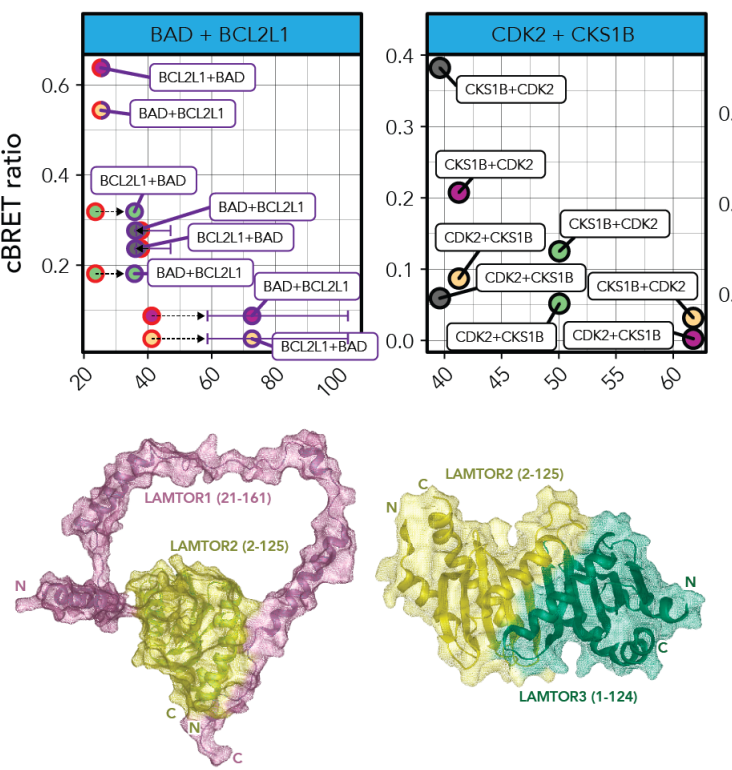

Based on PQB structure 6 E

LAMTOR2 (124/125 amino acids covered = 99\%)

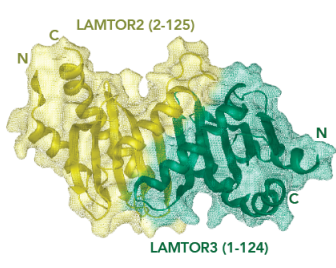

Based on PDB structure 6EHR
LAMTOR2 124125 amino acid LAMTOR3 124/124 amino acids covered = 1000
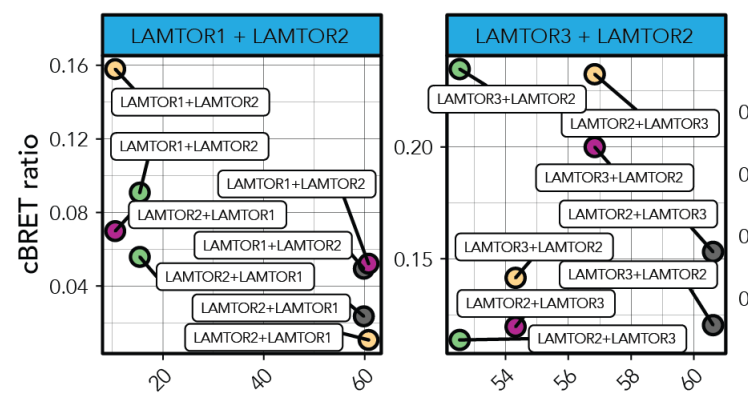
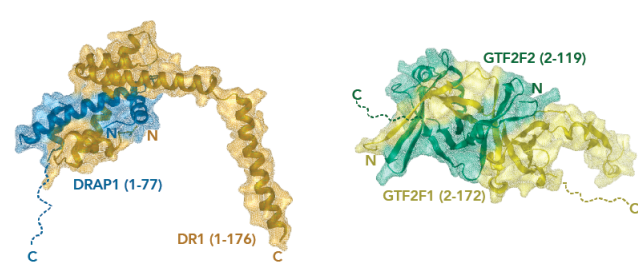

Based on PDB structure 1 HF
DRP (176/176 amino acids can

DRAP1 $977 / 205$ amino acids covered -3

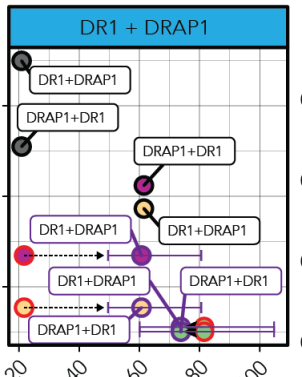

istance $[\AA ̊]$

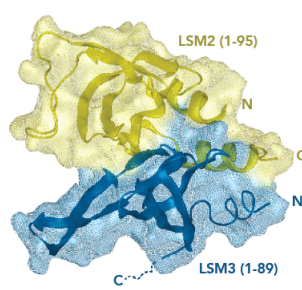

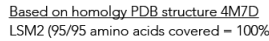

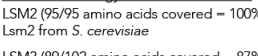

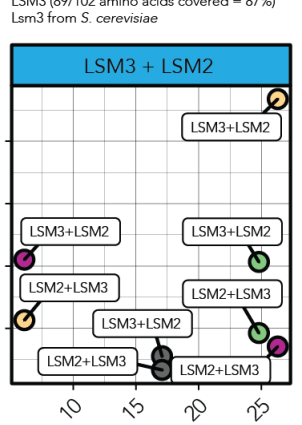

distance $[\AA]$

Based on PDB structure 1F3U
GTF2F1 (171/517 amino acids covered $\left.-33^{\circ}\right)$ GTF2F2(118/249 amino acids covered - $47 \%$

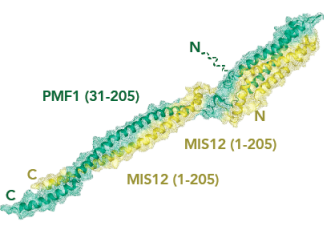

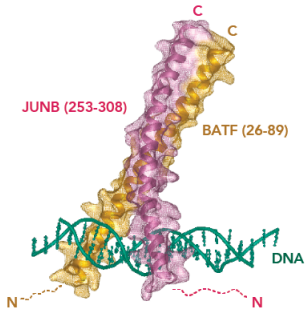
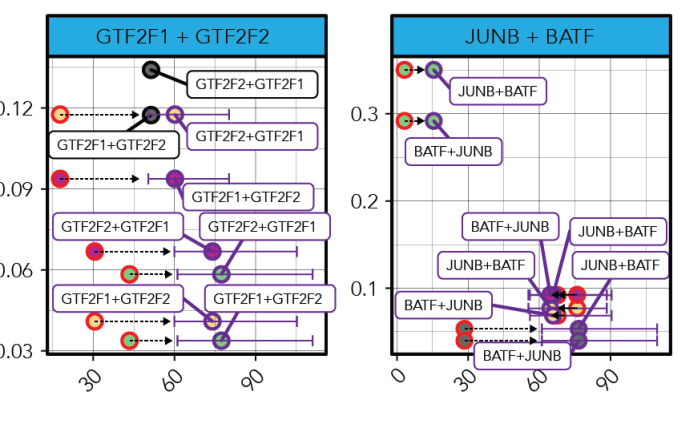

$\stackrel{\circ}{\longrightarrow} \longrightarrow \bigcirc_{100 \%}$

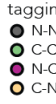

RRAGA (183-313)

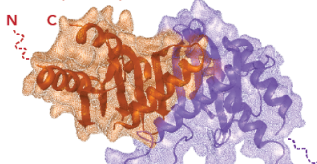

Ostructure-based reference RAAGC (239-399) \& 2

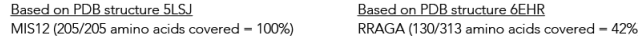
PMF1 (174/205 anino acids covered $=85 \%) \quad$ RRAGC 160/399 amino acids covered $=40 \%$
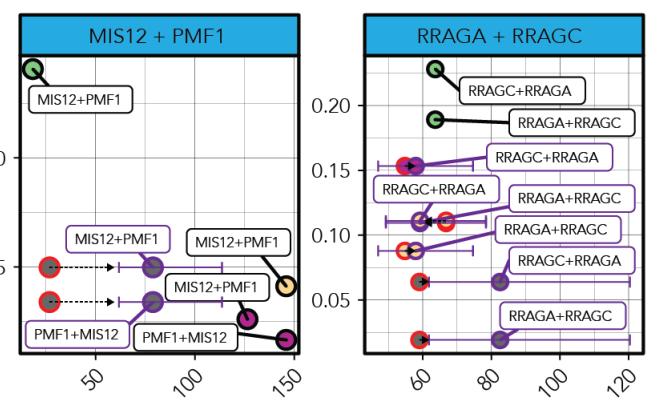
945 Supplementary Figure 10 (related to Figure 4). Subset of interactions where at least six out of the eight

946 tested tagging configurations scored positive in the LuTHy-BRET assay.

947 The following 3D structures are represented: 1G5J (BAD-BCL2L1), 1BUH (CDK2-CKS1B), 1JFI (DR1-DRAP1),

948 1F3U (GTF2F1-GTF2F2), 1A02 (JUNB-BATF), 6EHR (LAMTOR1-LAMTOR2 and LAMTOR3-LAMTOR2), 4M7D

949 (LSM3-LSM2), 5LSJ (MIS12-PMF1), and 6EHR (RRAGA-RRAGC). On each structure, the protein termini are

950 indicated by $\mathrm{N}$ or $\mathrm{C}$. Protein sequence coverages used in the structures are also indicated (\%). Protein regions that

951 are missing in the structures are indicated by dotted lines. For all interactions, cBRET ratios are plotted against the

952 molecular distances measured in the corresponding 3D structures or interpolated using the cBRET standard curve.

953 The quantitative scores outlined in black correspond to tagging configurations where the tagged protein termini are

954 resolved in the structures. These scores were used as reference data points for distance interpolations. Interaction

955 scores and structure-based molecular distances for tagging configurations where the tagged protein termini are

956 absent from the 3D structure are outlined in red. Interaction scores outlined in purple correspond to cBRET ratios

957 against interpolated molecular distances. Changes in structurally measured distances versus the interpolated

958 distances are indicated by dotted arrows. The purple horizontal error bars correspond to the 95\% confidence

959 intervals of the interpolated molecular distances. Tagging configurations are color-coded: N-N (grey), C-C (green),

$960 \mathrm{~N}-\mathrm{C}$ (purple), and C-N (yellow). Each data point on the graph is labeled (blank frame) according to the associated

961 tagging configuration tested: the protein indicated first is tagged with NanoLuc (NL) luciferase, while the second

962 protein is tagged with PA-mCitrine (PA-mCit) (e.g. DR1-DRAP1 (N-N, grey) corresponds to NL-DR1/PA-mCit-

963 DRAP1). Tagging configurations where tags are fused to the missing termini in the current 3D structure for one of

964 the two proteins are outlined in red (e.g. DR1-DRAP1 (N-C, purple) corresponds to NL-DR1/DRAP1-mCit-PA). The

965 size of the dots indicates the average percentage of protein sequence coverage in the corresponding 3D structure

966 of the PPI. 


\section{APPENDIX}

968 Supplementary Table 1: List of the multiprotein complexes meeting the prioritization criteria in this study.

969 Supplementary Table 2: Number of inter-complex pairs, direct interactions and indirect associations for the three

970 selected protein complexes: LAMTOR, BRISC and MIS12 as defined by their 3D structures. The numbers of

971 separate homodimer interactions not reported in the structures are also indicated.

972 Supplementary Table 3:

$973 \quad$ Sheet 1 - training sets: Positive and negative training sets for LuTHy cluster analysis.

974 Sheet 2 - clustering results: Supervised classification of direct and not-direct interaction clusters.

975 Supplementary Table 4: Surface and interface areas $\left(\AA^{2}\right)$ between directly interacting proteins in the LAMTOR,

976 BRISC and MIS12 complexes.

977 Supplementary Table 5: Names of protein complexes, protein subunits, and PDB IDs used in this study. The 978 amino acid sequences of the proteins used for binary PPI mapping or in the PDB structures are indicated.

979 Supplementary Table 6. Characterization of protein family (pfam) domains.

980 Supplementary Table 7: Structure-based and BRET-interpolated distances for the HTT-HAP40 interaction, and

981 for the intramolecular HTT sensors for HTTQ23 and HTTQ145.

982 Supplementary Table 8: Primer sequences used in this study.

983 Supplementary Table 9: Statistical reports for relevant figures.

984

985 Source Data Figures 1-2: Raw LuTHy data for interactions in hsPRS-v2.

986 Source Data Figures 3-5:

Sheet 1 - Multi-protein complexes: Raw LuTHy and mN2H mapping data for the selected multiprotein complexes. Molecular distances measured between structurally supported interactions between protein pairs within the LAMTOR, BRISC and MIS12 complexes.

Sheet 2 - hsPRS-PDB: Molecular distances measured between structurally supported heterodimer interactions of the hsPRS-v2 (hsPRS-PDB). 